\title{
The Gran Telescopio Canarias OSIRIS Broad Band First Data Release
}

\author{
M. Cortés-Contreras, ${ }^{1,2 \star}$ H. Bouy ${ }^{3}$ E. Solano, ${ }^{1,2}$ M. Mahlke, ${ }^{1,2}$ F. Jiménez-Esteban, ${ }^{1,2}$ \\ J.M. Alacid ${ }^{1,2}$ and C. Rodrigo ${ }^{1,2}$ \\ ${ }^{1}$ Departmento de Astrofísica, Centro de Astrobiología (CSIC-INTA), ESAC Campus, Camino Bajo del Castillo s/n \\ E-28692 Villanueva de la Cañada, Madrid, Spain \\ ${ }^{2}$ Spanish Virtual Observatory, Spain \\ 3 Laboratoire d'astrophysique de Bordeaux. Université de Bordeaux. CNRS, B18N Allée Geoffroy Saint-Hilaire, 33615 Pessac, France.
}

Accepted XXX. Received YYY; in original form ZZZ

\begin{abstract}
We present the first release of GTC OSIRIS Broad Band data archive. This is an effort conducted in the framework of the Spanish Virtual Observatory to help optimize science from the Gran Telescopio Canarias Archive. Data Release 1 includes 6788 broad-band images in the Sloan griz filters obtained between April 2009 and January 2014 and the associated catalogue with roughly 6.23 million detections of more than 630000 unique sources. The catalogue contains standard PSF and Kron aperture photometry with a mean accuracy better than 0.09 and $0.15 \mathrm{mag}$, respectively. The relative astrometric residuals are always better than 30 mas and better than 15 mas in most cases. The absolute astrometric uncertainty of the catalogue is of 0.12 arcsec. In this paper we describe the procedure followed to build the image archive and the associated catalogue, as well as the quality tests carried out for validation. To illustrate some of the scientific potential of the catalogue, we also provide two examples of its scientific exploitation: discovery and identification of asteroids and cool dwarfs.
\end{abstract}

Key words: techniques: image processing - Astronomical data bases: catalogues Astronomical data bases: virtual observatory tools

\section{INTRODUCTION}

Astronomical data archives and catalogues have become a new paradigm in the astrophysics research. Reduced (photometrically and astrometrically corrected images, spectra ready for immediate scientific exploitation,...) and high-level (catalogues, mosaics, stacked images,...) data products are of fundamental importance for archives as they enhance their use by the community. Moreover, these science-ready data products provide a higher visibility of the project results as clearly demonstrated by the large number of refereed papers based on archived data from projects like SDSS (York et al. 2000), 2MASS (Cutri et al. 2003), UKIDSS (Lawrence et al. 2007a) and WISE (Wright et al. 2010a), to name a few. By using these resources, astronomers are able to conduct research projects that would otherwise be very timeconsuming or completely unaffordable.

The Gran Telescopio de Canarias ${ }^{1}$ ( GTC), with its 36 individual hexagonal segments acting as a $10.4 \mathrm{~m}$ diameter

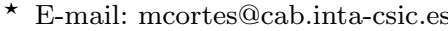

1 http://www.gtc.iac.es/
}

single mirror, is currently the largest optical-infrared telescope in the world. Operated by the Instituto de Astrofísica de Canarias, it is located at the Observatorio del Roque de Los Muchachos in La Palma (Spain), which provides excellent observing conditions.

OSIRIS (Optical System for Imaging and lowIntermediate-Resolution Integrated Spectroscopy) is an imager and spectrograph for the optical wavelength range, located in the Nasmyth-B focus of GTC. OSIRIS allows broadband imaging over a field of view of $7.8 \times 7.8$ arcmin unvignetted covering a spectral range from $\lambda 3650 \AA$ to $\lambda 10000 \AA$, with a high transmission coefficient, in particular at longer wavelengths. The spectral range is covered by the Sloan system broadband filters: u' ( $\lambda 3500 \AA)$, g' $(\lambda 4750 \AA)$, r' $(\lambda 6300 \AA)$, i' $(\lambda 7800 \AA)$, z' $(\lambda 9250 \AA)$. The filter tranmission and detector efficiency curves, taken from the Filter Profile Service maintained by the Spanish Virtual Observatory $^{2}$, are shown in Figure 1. More information on the OSIRIS capabilities can be found at the $G T C$ web page ${ }^{3}$.

2 http://svo2.cab.inta-csic.es/theory/fps/
3 http://www.gtc.iac.es/instruments/osiris/ 


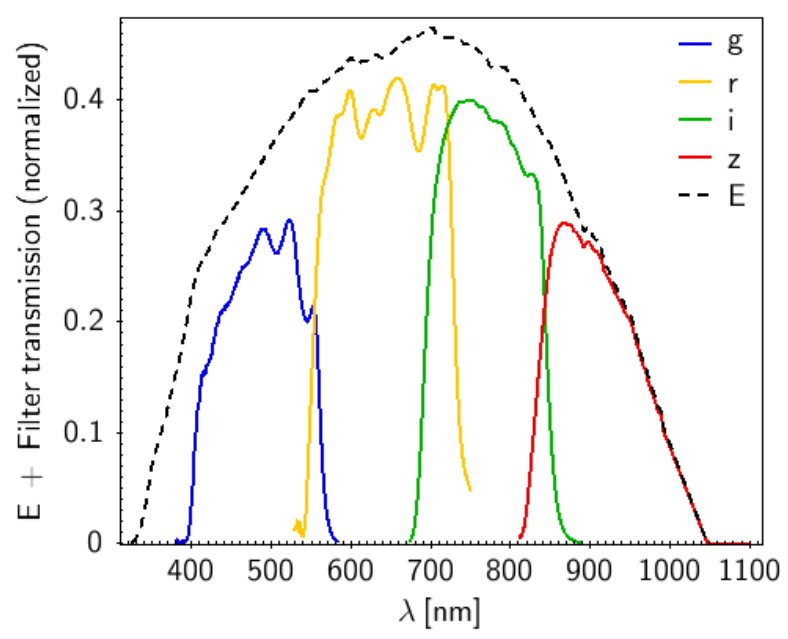

Figure 1. Transmission curves of the Sloan g' (blue), r' (yellow), i' (green) and z' (red) filters and the OSIRIS detection efficiency curve (dashed black).

The GTC archive $^{4}$ is in operation since 2011 and is hosted by the Spanish Virtual Observatory $\left(\mathrm{SVO}^{5}\right)$, one of the 21 national and trans-national Virtual Observatory initiatives distributed worldwide and coordinated by the International Virtual Observatory Alliance ${ }^{6}$.

This paper presents the first release of the OSIRIS Broad Band data. We begin in Sections 2 and 3 with a description of the data curation, preparation and processing of the images, followed by the source extraction in Section 4. We present astrometric and photometric calibrations in Sections 5 and 6. We then turn, in Section 7, to a description of the catalogue sources and a photometric validation exercise in which Pan-Starrs DR1 data are compared to OSIRIS observations. In Section 8, we introduce two science cases carried out with the catalogue as an attempt to show its potential for science exploitation. The paper ends with a description on how to access the data and a summary in Sections 9 and 10, respectively.

\section{DATA CURATION AND PREPARATION}

The first step consisted in the data selection and curation. Large facilities such as GTC produce vast amount of data coming from different instruments and observing modes. Since the goal of the present project is to deliver science grade products of the broad-band images, we therefore rejected spectroscopic observations and tunable filter observations taken with OSIRIS.

We obtain 27470 raw broad-band images from the $G T C$ Public Archive acquired between April 2009 and January 2014 in the griz bands. A total of 5638 among them were obtained using windowing (moving target images) and were discarded for the present release. The vast majority of them were targeting a few transiting targets requiring a high temporal frequency, and the loss in spatial coverage is minimum.

4 http://gtc.sdc.cab.inta-csic.es/

5 http://svo.cab.inta-csic.es/main/index.php

6 http://ivoa.net
Thus, it is important to remark that the absence of a source in the catalogue does not imply the absence of GTC OSIRIS observations in that position of the sky. To check this point, the GTC Archive must be used.

A significant number (13230) of early images were missing the standard MJD-OBS keyword giving the precise acquisition date, which is crucial for many studies focusing on the time-domain. Although it is possible to recover the missing information through other non-standard FITS keywords (e.g. OPENTIME), we will focus in this first data release on the the 8602 (27470 - 5638 - 13230) images with the standard MJD-OBS keyword. Among them, there are 858 calibration images that will not be included in the catalogue. Hence, we process 7744 OSIRIS images in the griz filters.

\section{DATA PROCESSING}

The individual raw images were processed using an upgraded version of Alambic (Vandame 2002), a software suite developed and optimized for the processing of large multiCCD imagers, which was adapted for OSIRIS. Alambic includes standard processing procedures such as overscan, bias and dark subtraction for each individual readout ports of each CCD, flat-field correction, bad pixel identification and masking, CCD-to-CCD gain harmonization, and fringing correction in the $z$-band. Alambic also combines the flatfield and bad pixel mask into a weight map for each individual dataset, and estimates the sky background in each image using an iterative multi-resolution median filtering. The method is very efficient, fast and robust for most stellar or extragalactic fields, but will produce artefacts for input images including large extended sources, as well as around the halo of very bright stars. Images and catalogues including large extended sources (i.e. covering a significant fraction of the field-of-view) should therefore be considered with caution, and probably reprocessed from scratch by the user.

Flat-fields were computed using the best twilight flatfield frames obtained over time windows of 15 days, since the OSIRIS flat-fields are considered to be very stable over periods of several weeks.

\section{SOURCE EXTRACTION}

Sources brighter than the $3-\sigma$ noise of the local background were detected and their photometry and position were measured using SExtractor (Bertin \& Arnouts 1996) and PSFEx (Bertin 2013). The weight-maps were used to properly modulate the detection threshold over the image. Three fixed apertures were used:

- 21,31 and 41 pixels in $1 \times 1$ binning mode, corresponding to $2.66,3.94$ and 5.21 arcsec, respectively

- 11,15 and 21 pixels in $2 \times 2$ binning mode, corresponding to $2.79,3.81$ and 5.33 arcsec, respectively.

In addition to these three fixed apertures, the Kron or automatic aperture as by SEXTRACTOR MAG_AUTO, the PSF (MAG_PSF) and the model (MAG_MODEL) photometry were also measured. A PSF model was fitted to the data with PSFEX using a second order polynomial to model the PSF variations 


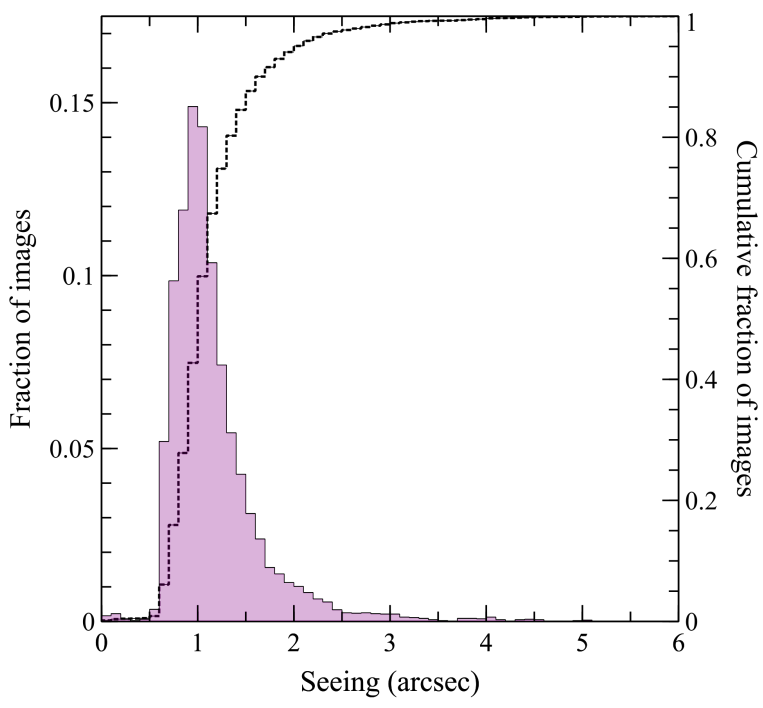

Figure 2. Distribution of average FWHM measured in the images themselves by PSFEx.

across the field-of-view. The PSF model was then used to fit every source using:

- standard PSF-fitting

- a two-dimensional Sérsic model convolved with the PSF model

The SExtractor model (i.e., PSF + Sérsic) parameters offer the advantage of being suited for both point-like and extended sources. Most galaxies are indeed resolved under sub-arcsecond seeing, and the PSF will not give a good fit.

Saturation was set at 62000 counts, even though the detector is expected to behave linearly up to the 16 bits encoding limit. This is an approximate value and, therefore, some saturated sources may still be present in the catalogue of extracted sources (see Section 7.1.1). For a better identification of saturated pixels we will use MAxiMAsk (Paillassa et al. 2019) in future data releases.

A few morphometric parameters were also extracted. SExtractor offers the possibility to measure the Fullwidth at half maximum (FWHM), the flux radius (defined as the circular aperture radius enclosing half the total flux), the elongation and the ellipticity. The model fitting also provides useful information about the morphometry and is particularly interesting for extended and saturated sources (Bouy et al. 2013). Figure 2 shows the distribution of FWHM as measured by PSFEx. The distribution peaks around 1 arcsec and more than half of the images were obtained under subarcsecond seeing. Note that the distribution extends far towards large values thanks to the $G T C$ filler program specifically designed to make use of poor ambient conditions.

The SExTRACTOR and PSFEx configuration files used to produce the catalogues, as well as the parameters obtained are given in Tables A1, A2 and A3.

\section{ASTROMETRIC CALIBRATION}

A precise astrometric calibration was computed using SCAMP (Bertin 2006). The 2MASS catalogue was used as reference for most frames, except when too few 2MASS sources were available in the field-of-view to derive a proper astrometric solution. In those cases, the SDSS (DR10) (Ahn et al. 2014) or USNO-B1 (Monet et al. 2003) catalogues were used. The absolute astrometric accuracy is therefore set by these catalogues and is expected to be better than 0.1 arcsec. Nonlinear geometric distortions were fitted using a third order polynomial, as illustrated in Fig. 3. The internal accuracy is estimated to be better than 30 mas in all cases, and better than 15 mas in most cases. Celestial coordinates are given at the observing epoch and Equinox J2000.0.

The astrometric calibration allowed us to detect 530 problematic images, typically including technical images with no sources at all, images obtained during a tracking failure or having suffered a read-out failure. The total number of remaining images added to 7214 , distributed over the entire northern sky accessible from La Palma, as illustrated in Fig. 4.

This figure reflects the patchy nature of the OSIRIS observations, with a modest fraction $(0.02 \%)$ of the full sky being covered $\left(8.05 \mathrm{deg}^{2}\right)$. This is a primary difference with typical survey projects covering a regular geometric pattern in the sky and with uniform properties, such as exposure time and filter set.

Individual astrometrically calibrated images can be easily downloaded from the $G T C$ archive and stacked to make a larger and/or deeper mosaic of a given field. Because the astrometric calibration was obtained using the AstrOmatic software suite, we recommend to use SWARP (Bertin et al. 2002) for the stacking. SWARP will indeed properly interpret the astrometric solution included in the image headers and the weight map delivered with every individual image by simply using the "-WEIGHT_TYPE MAP_WEIGHT" option.

\section{FLUX CALIBRATION}

The set of OSIRIS images used to build the catalogue constitute, by definition, a heterogeneous collection where the variety of observational parameters (e.g. exposure times) reflect the diversity of science cases carried out with them. While images with the shortest exposure times typically present bright sources and few or no faint objects, images with the largest exposure times, aimed at reaching fainter sources, usually include saturated objects. This wide range of exposure times reflects both the potential and the challenge of the associated catalogue.

We adopted the Pan-STARRS DR1 survey (PS1 hereafter) (Kaiser et al. 2010; Chambers et al. 2016) as the reference to photometrically calibrate GTC OSIRIS images. The PS1 catalogue includes PSF and Kron photometric measurements, which we will use to calibrate our MAG_PSF and MAG_AUTO photometry, respectively.

In order to obtain the calibration parameters, we searched for counterparts to each OSIRIS source in PS1 within 1.0 arcsec. If more than one counterpart exists in the search radius we took the nearest one. Each individual CCD subimage has been calibrated independently. For a source to be considered as a calibration source, it must fulfill the following requirements:

- PS1 sources must be point-like sources with PSF- 

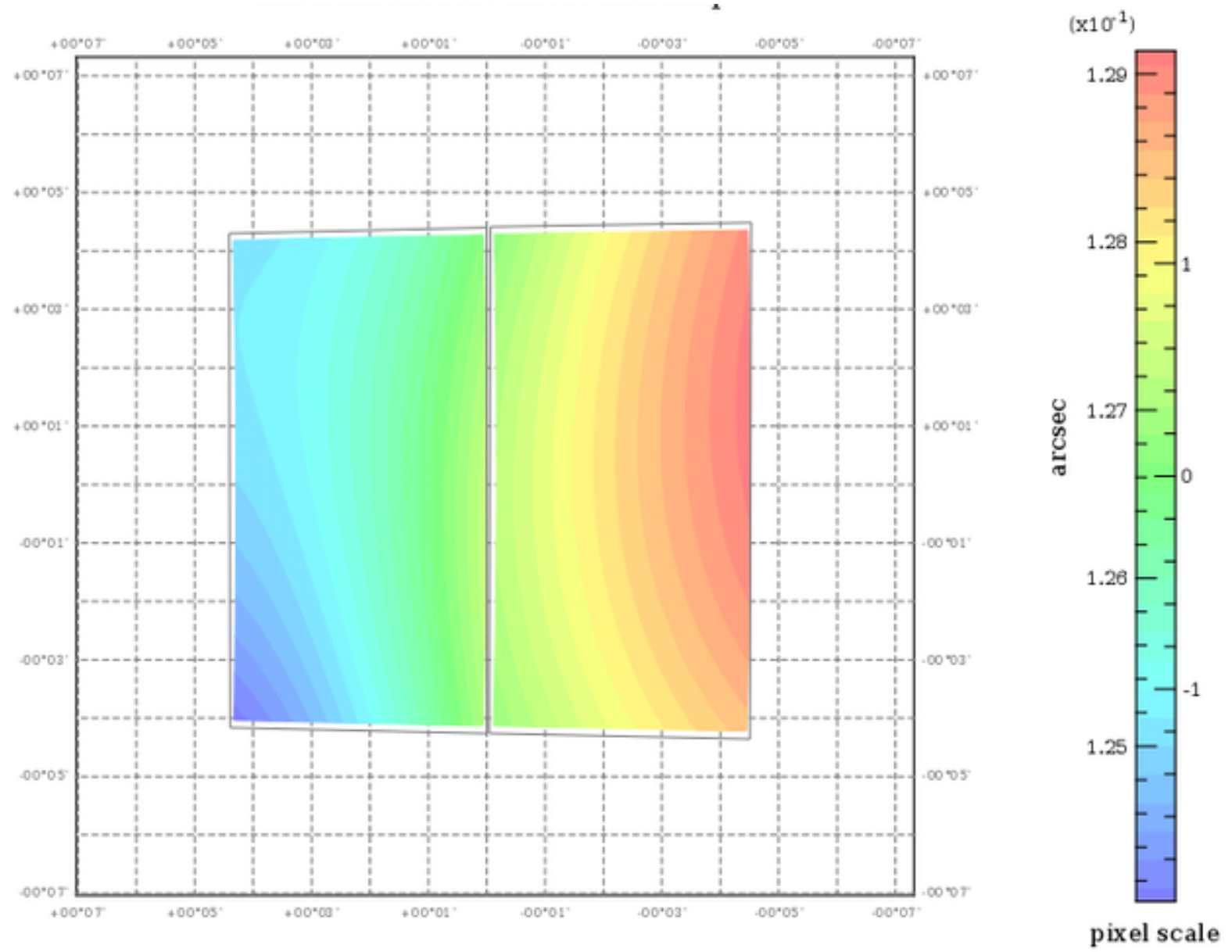

Figure 3. Distortion map for the $i$-band filter as measured by SCAMP.

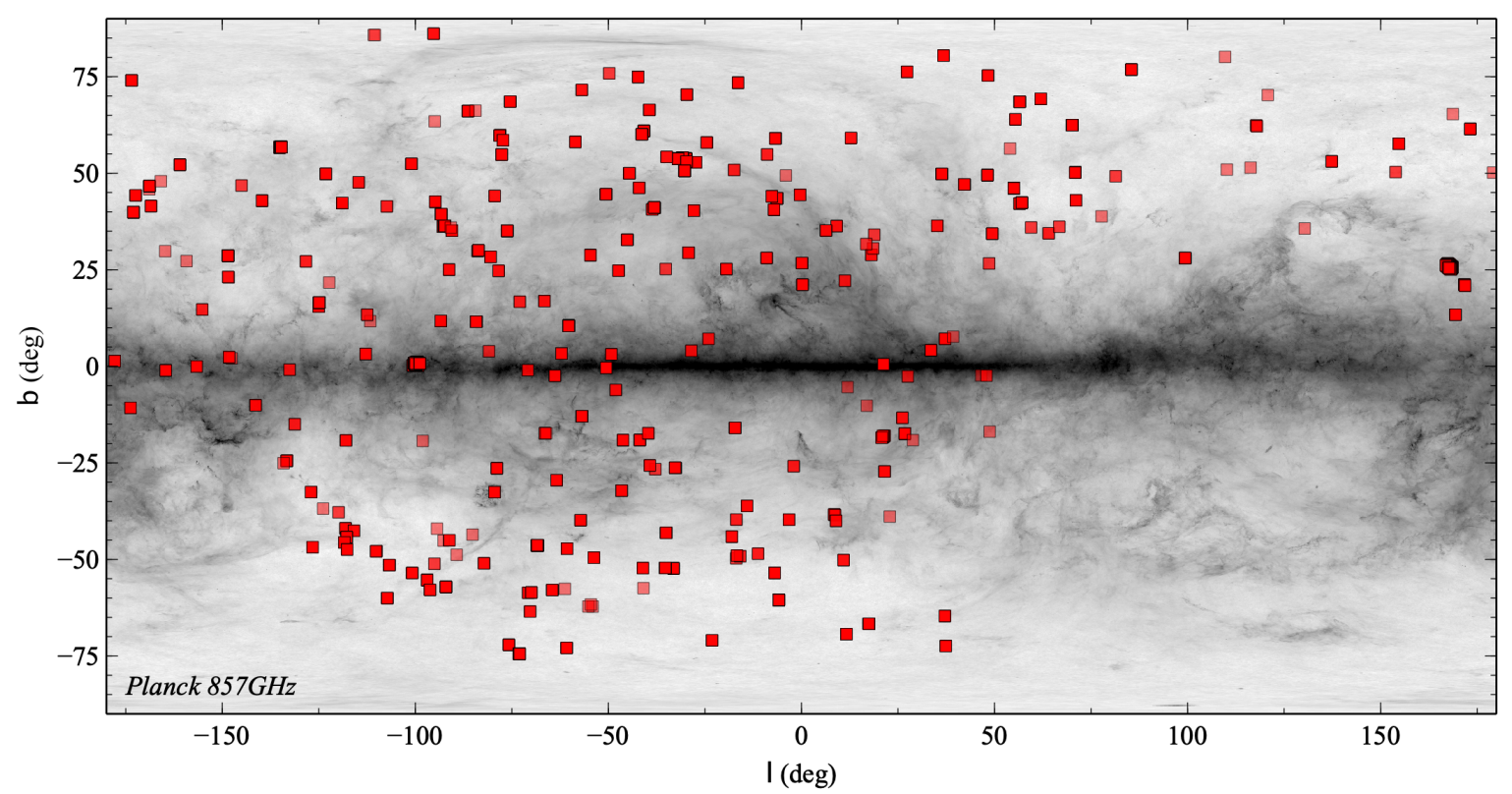

Figure 4. Location of the 7214 OSIRIS pointings overplotted on an all-sky $857 \mathrm{GHz}$ Planck map. 
Kron $<0.05$ mag (Chambers et al. 2016), and Qual flag equal to 52 .

- PS1 sources must be within the saturation and detection limits of the survey as stated in Chambers et al. (2016).

- Magnitude errors, both in PS1 and OSIRIS, must be smaller than 0.2 mag.

- In order to avoid spikes and extended sources, we select in our catalogue sources whose FWHM, elongation and ellipticity do not significantly deviate from the median value of all sources in the subimage.

With the selected sources, we carried out a sigmaclipping linear fit in each subimage, after which we performed the calibration only if there were more than six calibrating stars satisfying the criteria listed above. Besides, we provide calibrated magnitudes only if the Pearson correlation coefficient $(r)$ of the linear fit is greater than 0.98 . Of the 14428 subimages available $(7214 \times 2)$, we calibrated 13555 and 13403 in MAG_PSF and MAG_AUTO photometry, respectively. Of them, 12454 and 11614 have a correlation coefficient $r \geq 0.98$ ( $82 \%$ and $87 \%$, respectively). A total number of 11045 subimages have both, PSF and automatic photometric calibrations with $r \geq 0.98$. The differences in the number of calibrated subimages arise, on one hand, from the discrepancies in magnitude errors in PSF and AUTO photometry, which prevent the same detection to pass the magnitude cuts in both photometries. On the other hand, the different procedures of flux measurements by SEXTRACTOR in few cases lead to a wrong measure of the FLUX_PSF but not of FLUX_AUTO for the same source and viceversa. We could therefore obtain a varying number of sources in the same subimage for PSF and AUTO calibrations. The fact that the OSIRIS observations lie within the Pan-STARRS footprint ensures that, in all cases, the lack of photometric calibration is not due to a different spatial coverage of the two surveys but to the absence of sources fulfilling the criteria listed above or to the bad quality of the fit.

Figure 5 shows the differences between PS1 and OSIRIS calibrated magnitudes of the calibration sources as a function of PS1 magnitudes and the distributions of the absolute values of the magnitude differences taken from the 12454 and 11614 subimages with good linear fit in PSF (top first and second panels) and AUTO (third and fourth panels) photometry, respectively. The few number of outliers existent in the first and the third panels, typically associated to sources with larger magnitude errors that pass the criteria listed above and that remain after the linear fit within $3 \sigma$, have a minor impact in the calibration as we are using a weighted linear fit where the weight is inversely proportional to the error in magnitude. We show in the first and third panels the average magnitude differences in bins of $0.2 \mathrm{mag}$. Only bins with more than ten points are shown. In all bands, these values are larger for faint sources (over $\sim 21.5 \mathrm{mag}$ in $g, r$ and $i$ bands, and over $\sim 20.0 \mathrm{mag}$ in the $z$ band). We also observe an increasing trend towards the bright edge of the plots. This behaviour is observed in magnitude intervals with less than 100 sources, very few compared to the several thousands included at intermediate magnitude bins. These averaged differences of magnitudes remain under $0.1 \mathrm{mag}$ in PSF and under $0.2 \mathrm{mag}$ in AUTO comparisons for all bands. On average, AUTO photometry presents larger scatter compared with PSF. Mean magnitude absolute dif- ferences (second and fourth panels) are marginal. They vary from 0.063 to $0.079 \mathrm{mag}$ in PSF photometry and from 0.096 to $0.098 \mathrm{mag}$ in AUTO photometry, depending on the filter.

The difference in the filter transmission curves of the PS1 and OSIRIS filters do also contribute to the discrepancy between magnitudes. Figure 6 shows the comparison between the transmission curves for each filter. In both cases, the throughput of the instrument is considered. In the case of the PS1 curves, taken from the SVO Filter Profile Service, we removed the contribution of the atmosphere, for comparison with OSIRIS' curves. While the $g$-band filters match fairly well, OSIRIS includes redder wavelengths in the $r, i$ and $z$ bands. To assess these differences in the calibrated photometry, we address a colour dependence test in Section 7.2.2.

The number of calibration sources per subimage ranges from 7 to 1510 for MAG_PSF and from 7 to 1323 for MAG_AUTO, with a mean number of 68 and 65 stars, and a standard deviation of 113 and 107, respectively.

The contribution to the total error budget of the calibration errors is, on average, between $0.05 \mathrm{mag}$ and $0.07 \mathrm{mag}$ in MAG_PSF and between $0.09 \mathrm{mag}$ and $0.12 \mathrm{mag}$ for $z$ in MAG_AUTO, depending on the filter.

Figure 7 shows the exposure time distributions of the calibrated subimages in MAG_PSF and MAG_AUTO photometry. In the whole catalogue, exposure time ranges from 0.5 to $900 \mathrm{~s}$. From the mean value of the distributions $(\sim 100 \mathrm{~s})$ we can estimate the mean limiting magnitudes of the catalogue at 24.4, 23.9, 23.3, $22.1 \mathrm{mag}$ at $\mathrm{S} / \mathrm{N}=5$, and 23.6, 23.1, 22.6, $21.4 \mathrm{mag}$ at $\mathrm{S} / \mathrm{N}=10$ for $g, r, i$ and $z$ respectively. However, for the longest exposures, these magnitudes can reach 25.8 , 24.1, 23.6 and 22.4 magnitudes for griz at $\mathrm{S} / \mathrm{N}=5$.

To assess the photometric quality of our calibrations, we selected all calibration sources with magnitudes in the range $(18.5,18.5,18.5,18.0)<(\mathrm{g}, \mathrm{r}, \mathrm{i}, \mathrm{z})<(22.0,22.0,22.0,21.5)$ and with at least five detections with PSF and AUTO photometry, separately. These intervals of magnitudes were chosen in order to get a representative sample of the sources used for the calibration (over $90 \%$ for each filter and photometry), and to avoid saturation and very faint sources. We obtained the standard deviations of the measured magnitudes for each of the repeated sources. The median of the standard deviations in each filter are $\left(\sigma_{g}, \sigma_{r}, \sigma_{i}, \sigma_{z}\right)=(0.034,0.026,0.038,0.034)$ magnitudes for PSF and $\left(\sigma_{g}, \sigma_{r}, \sigma_{i}, \sigma_{z}\right)=(0.043,0.038,0.053,0.056)$ magnitudes for AUTO photometry. These low values reflect the good performance of the calibration parameters over time despite the assorted character of the observations.

We summarize in Table 1 the detailed numbers of the associated contribution of the calibration to the photometric errors, the range of exposure time, the mean limiting magnitudes at $\mathrm{SNR}=5$ and $\mathrm{SNR}=10$, and the typical magnitude deviations for the repeated sources in the above defined sample, for each filter. 

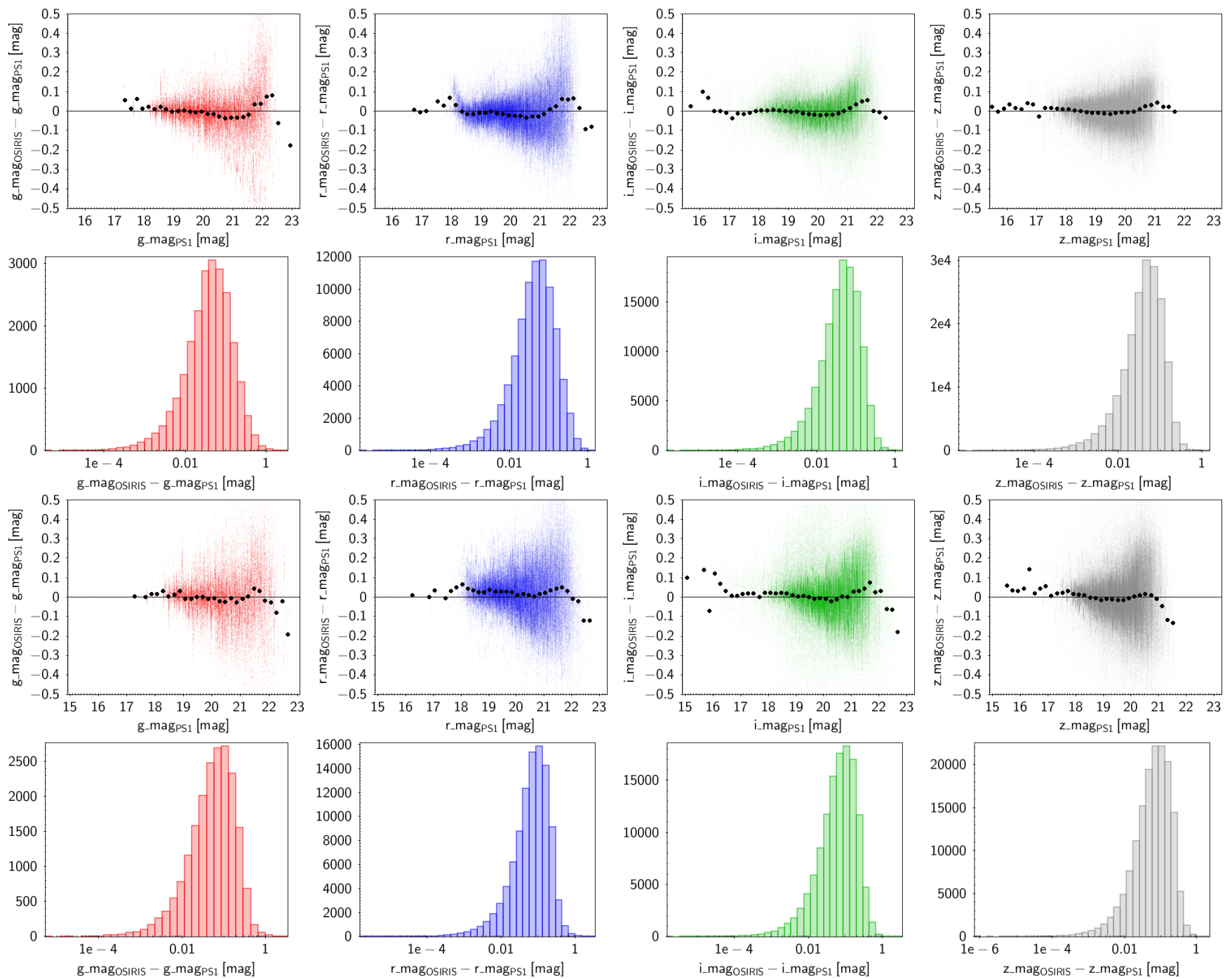

Figure 5. Comparison between PS1 and OSIRIS magnitudes of the sources used for the PSF (top first and second panels) and AUTO (third and fourth panels) photometric calibrations with $r>=0.98$. Black filled circles represent the average difference of magnitudes in bins of 0.2 mag. Only bins with more than ten sources are represented.

\section{CATALOGUE SOURCES}

\subsection{Construction}

\subsubsection{Removal of spurious and saturated detections}

To build the cleanest possible catalogue, we first removed sources with PSF instrumental magnitude errors equal to zero, larger than $1 \mathrm{mag}$, or equal to 99 (meaning that SExTRACTOR PSF fit did not converge). We also discarded sources with the SEXTRACTOR keywords FLUX_MAX, FLUX_RADIUS (defined as the half-light radius in Section 4) and FWHM_IMAGE smaller than or equal to zero, FLAGS_WEIGHT (weighted extraction flag related to the presence of close neighbours bright enough to significantly bias the photometry, bad pixels, blended objects, saturated pixels or other features) equal to two (note that we do not impose any condition on the extraction flags FLAGS parameter), and SNR_WIN (the window-based signal-to-noise ratio) smaller than five or equal to $1 \mathrm{e} 30$ (the latter, related to a bad extraction of the source). The limit in SNR_WIN at five is set to avoid sources with very poor photometry.

Although a saturation limit was set at 62000 counts when extracting the sources from the images, few saturated sources remain. Therefore, for each subimage in the catalogue, we removed saturated sources as well as bad pixels, cosmic rays and artifacts by accounting for the linear relation between the flux at the peak of the distribution (FLUX_MAX) and the integrated flux (FLUX_PSF), as shown in Figure 8. The procedure to remove spurious sources on each subimage consisted of running an iterative process that discards first all detections which ratio FLUX_MAX/FLUX_PSF deviates by more than $2 \sigma$ from the mean value. With the mean and standard deviation values of the remaining detections, we define a critical value of FLUX_MAX/FLUX_PSF equal to the mean value plus $3 \sigma$. From the original catalogue of sources of the subimage, we removed all detections which flux ratio is greater than this critical value. The rejected detections are represented with blue points in Figure 8 . 

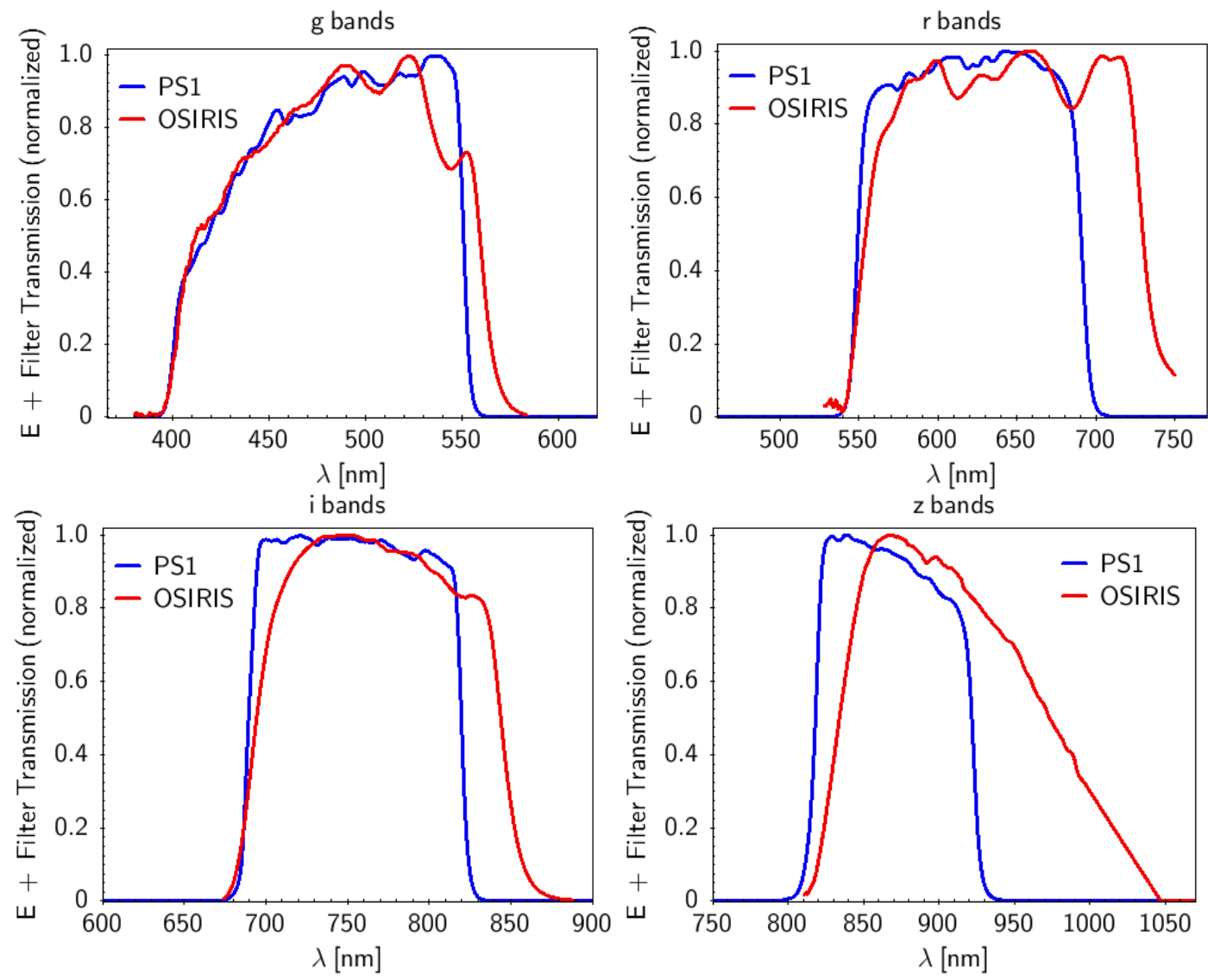

Figure 6. Comparison between the transmission curves of OSIRIS and PS1 in each band.

Table 1. Summary of the photometric properties of the calibrated subimages.

\begin{tabular}{|c|c|c|c|c|c|c|c|c|}
\hline & \multicolumn{4}{|c|}{ PSF } & \multicolumn{4}{|c|}{ AUTO } \\
\hline & $g$ & $r$ & $i$ & $z$ & $g$ & $r$ & $i$ & $z$ \\
\hline $\begin{array}{l}\text { Contribution to the } \\
\text { photometric error (mag) }\end{array}$ & 0.06 & 0.07 & 0.05 & 0.05 & 0.12 & 0.012 & 0.09 & 0.10 \\
\hline Exposure times (s) & $1-900$ & $1-360$ & $0.5-360$ & $0.5-300$ & $1-900$ & $1-542$ & $0.5-360$ & $0.5-30$ \\
\hline $\begin{array}{l}\text { Mean limiting magnitudes } \\
\text { at } \mathrm{SNR}=5 \text { (mag) }\end{array}$ & 24.4 & 23.9 & 23.3 & 22.1 & & & & \\
\hline $\begin{array}{l}\text { Mean limiting magnitudes } \\
\text { at } \mathrm{SNR}=10(\mathrm{mag})\end{array}$ & 23.6 & 23.1 & 22.6 & 21.4 & & & & \\
\hline $\begin{array}{l}\text { Limiting magnitudes for the } \\
\text { longest exposures and } \mathrm{SNR}=5 \text { (mag) }\end{array}$ & 25.8 & 24.1 & 23.6 & 22.4 & & & & \\
\hline & & & & Photomet & ic quality & & & \\
\hline Magnitude interval (mag) & $18.5-22.0$ & $18.5-22.0$ & $18.5-22.0$ & $18.0-21.5$ & $18.5-22.0$ & $18.5-22.0$ & $18.5-22.0$ & $18.0-21.5$ \\
\hline Median of the std. dev. $\sigma$ (mag) & 0.034 & 0.026 & 0.038 & 0.034 & 0.043 & 0.038 & 0.053 & 0.056 \\
\hline
\end{tabular}

Once the removal of spurious sources has been completed, we removed saturated sources by identifying the position in the relation FLUX_MAX vs. FLUX_PSF at which the detector breaks linearity. To determine this position, we iteratively performed a linear fit starting with sources with the lowest FLUX_MAX and increasing towards higher values of FLUX_MAX. We consider that linearity starts to fail when the Pearson correlation coefficient $r$ is lower than 0.98 and keeps decreasing. When there were no data available, we decreased the correlation coefficient to 0.96 . We then selected the upper half of the sample with highest FLUX_MAX to avoid non-linear behaviour at low fluxes (i.e., faint sources) and followed two complementary approaches:

- On one hand, we took the maximum number of sources 


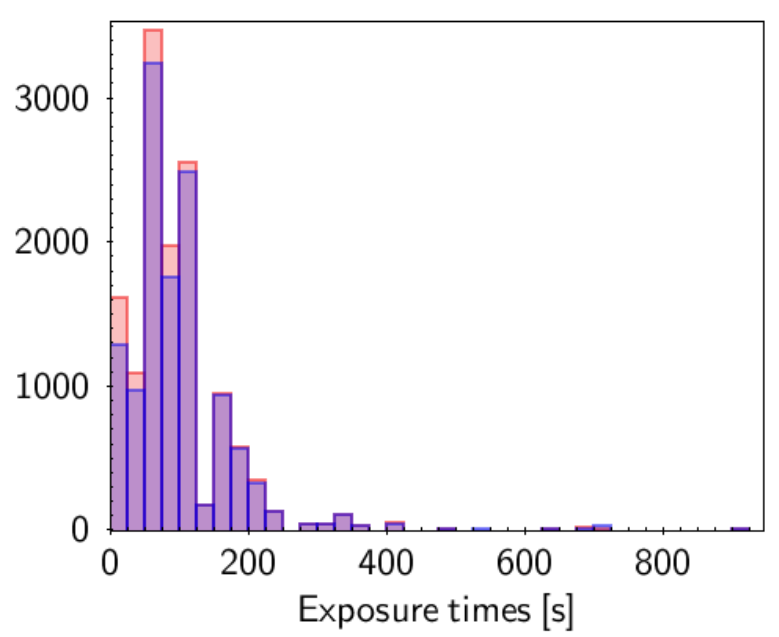

Figure 7. Histogram of the exposure times of MAG_PSF (red) and MAG_AUTO (blue) calibrated subimages with $r>=0.98$.

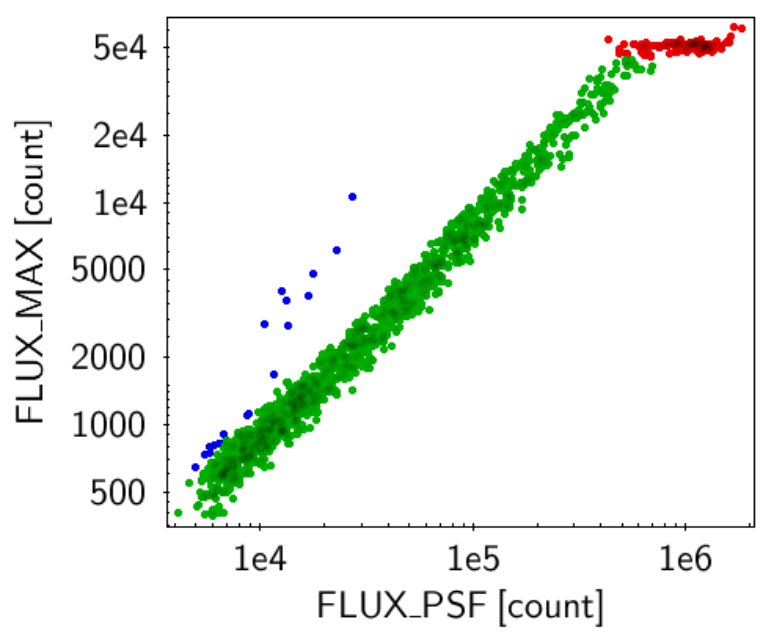

Figure 8. Maximum flux at the peak (FLUX_MAX) versus FLUX_PSF in logarithmic scale for detections in one subimage. Blue points represent spurious detections, red points represent saturated sources and green points stand for valid detections.

which linear fit provides a correlation coefficient greater than 0.98 (or 0.96 if it is the case). Since this condition itself does not ensure the removal of all saturated sources, we defined a subset with the detections above that maximum number (i.e., sources with higher flux) and established a cut in FLUX_MAX defined as the minimum value of the FLUX_MAX in the subset minus its standard deviation.

- On the other hand, we looked for the position at which the slope of the fit starts to decrease and defined a subset with detections starting at that point and with increasing fluxes. We again established a cut in FLUX_MAX defined as the minimum value of the FLUX_MAX in the subset minus its standard deviation. When the standard deviation was smaller than 2000 counts, we subtracted twice the value of the standard deviation to ensure the removal of all controversial sources.

In both cases, we removed all detections with FLUX_MAX above those limits.

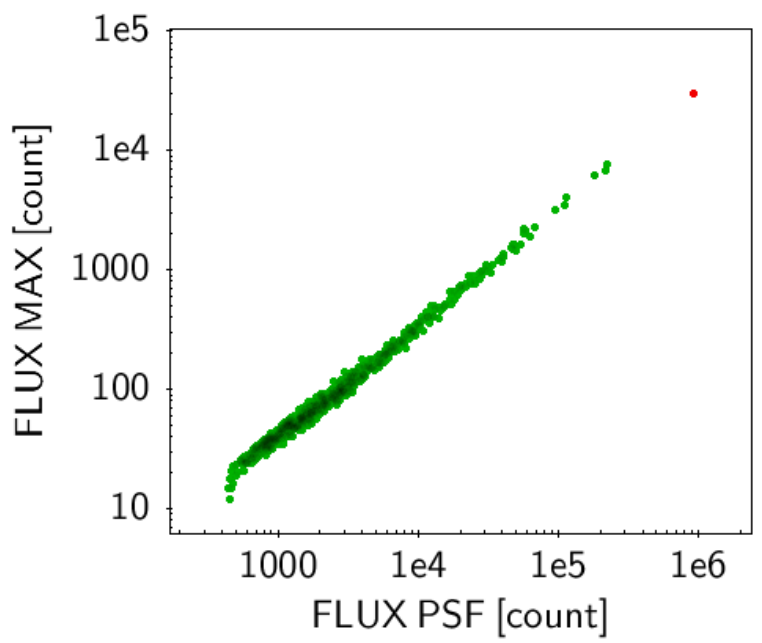

Figure 9. Maximum flux at the peak (FLUX_MAX) versus FLUX_PSF in logarithmic scale for detections in one subimage after the removal of spurious and saturated detections following the first two steps. Green points represent valid non-saturated source detections and the red point stands for a saturated source that follows the linear behaviour between fluxes and that accounts with more than 9000 counts in FLUX_MAX with respect to the maximum flux of the brightest-non saturated source in the subimage.

Despite the completion of these steps, we still found a few saturated sources that happen to follow the linear relation between fluxes as shown in Figure 9 and, therefore, have not been automatically removed. We observed that saturated sources have fluxes (FLUX_MAX) more than $\approx 9000$ counts higher than the corresponding flux to the brightest-non saturated source in the subimage. Hence, we removed detections which FLUX_MAX is at least 9000 counts greater than the brightest, non-saturated source in the subimage.

The above described procedure does only relate to PSF photometry. Hence, in parallel to this, we built a second catalogue considering exclusively calibrated MAG_AUTO magnitudes and following similar steps than before. The only difference comes from the identification of saturated sources, because the relation between FLUX_MAX and FLUX_AUTO displays a higher dispersion and, therefore, executing the procedure to remove saturated sources as described above prevented us from obtaining a complete catalogue in AUTO photometry, since sources in more than $50 \%$ of the calibrated subimages did not fulfill the required criteria. Therefore, for each subimage, we took the cuts in FLUX_MAX determined when removing saturated sources from PSF photometry and eliminated all sources in this second catalogue with FLUX_MAX higher than those values. This approach limits the AUTO photometry to the 11045 subimages that have both, PSF and AUTO calibrated photometry.

Moreover, we included a photometric flag to each detection in the catalogue to account for magnitudes beyond the magnitude coverage of the PS1 sources used in the photometric calibration in PSF (Flag_psf) and AUTO (Flag_auto) photometry. "B" and "C" stand for magnitudes fainter and brighter than the magnitude coverage of PS1, respectively. Detections with magnitudes in between are flagged with "A". 


\subsubsection{Morphologic classification}

In a second step, we evaluated in our catalogue the magnitude difference limit PSF - AUTO at $0.05 \mathrm{mag}$ proposed in the PS1 catalogue (Farrow et al. 2014) to separate pointlike (PSF - AUTO < $0.05 \mathrm{mag}$ ) and extended sources (PSF - AUTO > $0.05 \mathrm{mag}$ ). Of the OSIRIS detections in the $g$ band under $21 \mathrm{mag}$ (limit above which the high dispersion makes this classification unreliable) and over $\sim 17 \mathrm{mag}$ (magnitude limit imposed by PS1) with PS1 counterparts and that satisfy PS1 gmag - gKmag < 0.05 mag (i.e., point-like sources), $95.5 \%$ were also classified as point-like sources following the same criterion with OSIRIS photometry (i.e., had PSF - AUTO $<0.05 \mathrm{mag}$ ). Hence, we could consider this photometric criterion to be valid to select point-like sources. However and due to its magnitude restriction, we do not ascribe an extended or point-like source flag in the catalogue following this rule. We evaluated instead the separation between point-like and extended sources at any magnitude range by looking into the FWHM_IMAGE, elongation and ellipticity parameters. To do so, we selected sources in each subimage with PSF calibrated magnitudes between 17 and $21 \mathrm{mag}$ (regardless the filter) and with magnitude differences PSF - AUTO $<0.05 \mathrm{mag}$, and compute their FWHM_IMAGE, elongation and ellipticity mean values and standard deviations. Sources in the subimages which parameter values differed by more than the mean value within $2 \sigma$ were tagged as extended sources and as point-like sources otherwise.

To assess the goodness of this criterion, we compared both, the OSIRIS photometric and morphometric classifications, in the interval between 17 and 21 mag. In all bands, more than $92 \%$ of the sources identified photometrically as point-like were also labeled as point-like using morphometric parameters. Moreover, extended sources were also in agreement in more than $75,83,74$, and 72 per cent of the cases in the $g, r, i$, and $z$ bands, respectively. We therefore conclude that the morphometric criterion can be extended to all detections in the catalogue, regardless their magnitudes, to tabulate them as point-like or extended sources.

For 1378 subimages in the four bands, we could not apply this selection because of the lack of automatic (Kron) magnitudes due to the absence of photometric calibration, or the lack of sources with magnitude differences under $0.05 \mathrm{mag}$ and magnitudes between 17 and $21 \mathrm{mag}$. Since the number of subimages without any classification was significantly large, we decided to use PS1 magnitudes to identify point-like sources and compute their FWHM_IMAGE, elongation and ellipticity mean and standard deviations values to be used as a reference for the classification of all detections in the subimage. In these cases, the classification is noted in the catalogue as " $\mathrm{P}$ *" for point-like and " $\mathrm{E}^{*}$ " for extended sources.

After this, there are still 77 subimages (39246 detections representing $0.6 \%$ of the catalogue) for which we were not able to ascribe any classification.

The privileged location and weather conditions of the Observatory favour astronomical observations with a typical seeing of 1 arsec and reaching often lower values. Detections with FWHM below 0.5 arcsec were removed as they were just simply artifacts. Detections with FWHM over 7 arcsec and/or with ellipticities over 0.7 were also removed. The mean ellipticity measured in point-like sources is $0.11 \pm 0.07$
Table 2. Number of subimages and detections per filter in the catalogue.

\begin{tabular}{ccc}
\hline \hline Filter & $\begin{array}{c}\text { Number of } \\
\text { subimages }\end{array}$ & $\begin{array}{c}\text { Number of } \\
\text { detections }\end{array}$ \\
\hline Sloan g & 1328 & 532760 \\
Sloan r & 2866 & 1585249 \\
Sloan i & 3825 & 2041184 \\
Sloan z & 4390 & 2067327 \\
\hline
\end{tabular}

Table 3. Number of sources detected in one to four bands.

\begin{tabular}{cc}
\hline \hline $\begin{array}{c}\text { Number of } \\
\text { bands }\end{array}$ & $\begin{array}{c}\text { Number of } \\
\text { sources }\end{array}$ \\
\hline & $19991(3.2 \%)$ \\
3 & $64350(10.2 \%)$ \\
2 & $106747(16.8 \%)$ \\
1 & $442471(69.8 \%)$ \\
\hline
\end{tabular}

with just a $1.9 \%$ showing an ellipticity worse than $e>0.3$. This confirms the good performance of the GTC tracking capability.

\subsubsection{Resulting catalogue}

The next step is to build a science-ready, user-friendly catalogue containing both astrometric and photometric information as well as flags to warm about quality issues.

In summary, the catalogue contains 6226520 detections corresponding to 633559 different sources in 12409 subimages. Detections were merged into sources by performing an internal match of the whole catalogue (this is, regardless the photometric band) within 0.5 arcsec using STILTS (Taylor 2006). This value is a trade-off between completeness and reliability. Larger values may allow unrelated detections to be linked in the same source while smaller values would pose problems for faint sources with large centroiding errors. A detailed description of each column in our detection catalogue is given in Table B1.

Table 2 lists, for each filter, the number of subimages and detections contained in the catalogue.

Table 3 summarizes the number of sources that have been detected in one, two, three or the four bands, regardless which bands are they. Near $30 \%$ of the sources in the catalogue has been detected in two or more bands and only $3.2 \%$ has been detected in the four bands.

Astrometric errors in the catalogue were computed from the quadratic sum of the windowing position errors (i.e., the errors in the ellipse parameters reported by SEXTRACTOR) and the estimated absolute astrometric calibration uncertainty mentioned in Section 5. The mean accuracy in the catalogue is 0.12 arcsec.

In order to provide accurate photometry at a single epoch, we identified the best detection for each source, hereafter refereed to as primary detection. The criteria used to select the best detection were to have signal to noise ratio 
above the mean value for that source, and the least relative PSF or AUTO magnitude error. A total of 1209058 primary detections satisfied the above criteria. Primary detections are flagged in the catalogue as $p$ while the rest of detections are flagged with $s$ under Flag_source.

In addition, we composed a source catalogue with these primary detections of each source, for the user to easily access the best photometry. It contains selected data such as the source identifier, equatorial coordinates, PSF and AUTO calibrated magnitudes with their corresponding flags, epochs and url of the associated image in each band, and a source class parameter $(c l)$ defined as the ratio between the number of detections classified as point-like ("P" or "P*") and the total number of detections. The source class parameter takes values between 0 and 1, being 0 when the source has always been identified as extended and 1 when it has always been identified as point-like.

This source catalogue is complementary to the previously defined one containing all detections, magnitudes and parameters. Table B2 contains a detailed description of each column in the source catalogue.

Typical saturation and limiting magnitudes in the catalogue in each filter are 13.1-24.6 mag in $g, 13.6-24.5 \mathrm{mag}$ in $r$, $13.1-23.9 \mathrm{mag}$ in $i$, and $12.0-22.7 \mathrm{mag}$ in $z$, respectively. Saturation magnitudes correspond to the minimum value of the PSF magnitude in the catalogue and limiting magnitudes correspond to the 90 th percentile, per each filter. This means that the magnitude limits in the OSIRIS catalogue are between 1.4 and 2 magnitudes brighter and between 0.4 and 1.4 magnitudes fainter than PS1, depending on the filter. Figure 10 shows a GTC OSIRIS image together with all PS1 and $G T C$ sources in the field of view. The increased depth of the OSIRIS catalogue (1378 objects) compared to PS1 (366 objects) is clearly seen. The catalogues have 208 sources in common. There are 158 sources that are in PS1 and not in our catalogue due to saturation or Flags_weight parameter equal to two (see the first paragraph in Section 7.1.1). Magnitudes of the 1170 sources in our catalogue that are not in PS1 range from 20.0 to $25.4 \mathrm{mag}$, which depending on the filters, would be beyond the magnitude limit of PS1.

Figure 11 represents averaged PSF and AUTO magnitude errors versus magnitude in bins of $0.5 \mathrm{mag}$, illustrating the sensitivity reached in the catalogue on each of the four filters. The trend is to increase with increasing magnitudes (i.e., towards fainter sources). In all bands, mean magnitude errors are under $0.1 \mathrm{mag}$ up to $21.5 / 19 \mathrm{mag}$ and do not exceed 0.30 / $0.35 \mathrm{mag}$, in PSF / AUTO photometry, respectively. In PSF photometry, the mean photometric accuracy of the catalogue is $0.09 \mathrm{mag}$ and $0.15 \mathrm{mag}$ in AUTO photometry.

\subsection{Catalogue quality assessment}

\subsubsection{Comparison with PS1}

Top panel in Figure 12 compares good quality PS1 sources (Qual equal to 52) versus OSIRIS PSF magnitudes. We observe good agreement up to PS1 $\sim 21.5 \mathrm{mag}$ in the $g$ and $r$ bands, $\sim 21.0 \mathrm{mag}$ in the $i$ band and $\sim 20.5 \mathrm{mag}$ in the $z$ band, magnitudes above which we observe a tendency towards fainter OSIRIS magnitudes. Most outliers in these plots do also missmatch when comparing their PS1 magni- tudes with clean (q_mode equal to '+') SDSS DR12 photometry (see bottom panel in this figure). Note that the number of sources represented in the plots of the bottom panel is lower than the number of sources represented in the plots of the top panel due to the requirement of having good PS1 and SDSS DR12 photometry. In the comparison of PS1 with SDSS DR12 we observe as well the tendency towards fainter magnitudes above 20-21.5 mag, depending on the filter. Hence, we ascribe these magnitude differences to an intrinsic feature of PS1 photometry.

Figure 13 shows, for each filter, the normalized cumulative distribution of the PSF magnitude absolute differences between OSIRIS and PS1 sources with good quality flags ( Qual equal to 52). For $90 \%$ of the sample of sources in the magnitude interval of linear behaviour, the photometric scatter in absolute values is below $0.16,0.19,0.18$, and 0.16 magnitudes in $g, r, i$ and $z$, respectively. This reflects the good photometric agreement between catalogues.

\subsubsection{Colour dependence}

To investigate the effect of a colour term in the photometric calibration, we compared the colour differences between OSIRIS and PS1 as a function of the calibrated magnitudes in the top panel of Figure 14. The large dispersion observed towards fainter sources is likely associated to the already noticed magnitude differences over 20-21.5 mag when comparing OSIRIS with PS1 (see Figure 12). Moreover, the number of sources with significantly high colour differences is not statistically representative (less than $1 \%$ of the plotted sample). The bottom panel of the figure shows the difference of magnitudes in the $\mathrm{z}$ band with respect to the PS1 $r-z$ (left) and $i-z$ colours (right). In light of these plots, we do not observe any colour dependence.

\subsubsection{Binning mode and pixel position dependence}

We verify whether the observed magnitude differences of sources with PSF magnitudes fainter than 20.5-21.5 mag (depending on the filter) when comparing with PS1 photometry have a relation with the pixel position of the source in the CCD.

Figure 15 shows, for each filter, the $x y$ pixel position of sources with PSF magnitudes fainter than $21.5 \mathrm{mag}$ in $g$ and $r, 21.0 \mathrm{mag}$ in $i$, and $20.5 \mathrm{mag}$ in $z$ (i.e., sources with the largest magnitude deviations) and with good quality PS1 photometry. OSIRIS standard observing mode uses $2 \times 2$ binned pixels, this is, images of $\sim 1024 \times 2048$ pixels size. This is the binning mode where the majority of the observations were performed. In general, binning the pixels aims to increase the signal to noise of measured images at a cost of losing spatial resolution. In light of the random arrangement of the sources in the CCD, we can conclude that there is no dependence of the magnitude differences either with the binning mode of observation or the pixel position.

\subsubsection{Internal photometric precision}

In order to estimate the internal photometric precision of the catalogue, we compared for each filter the magnitudes 


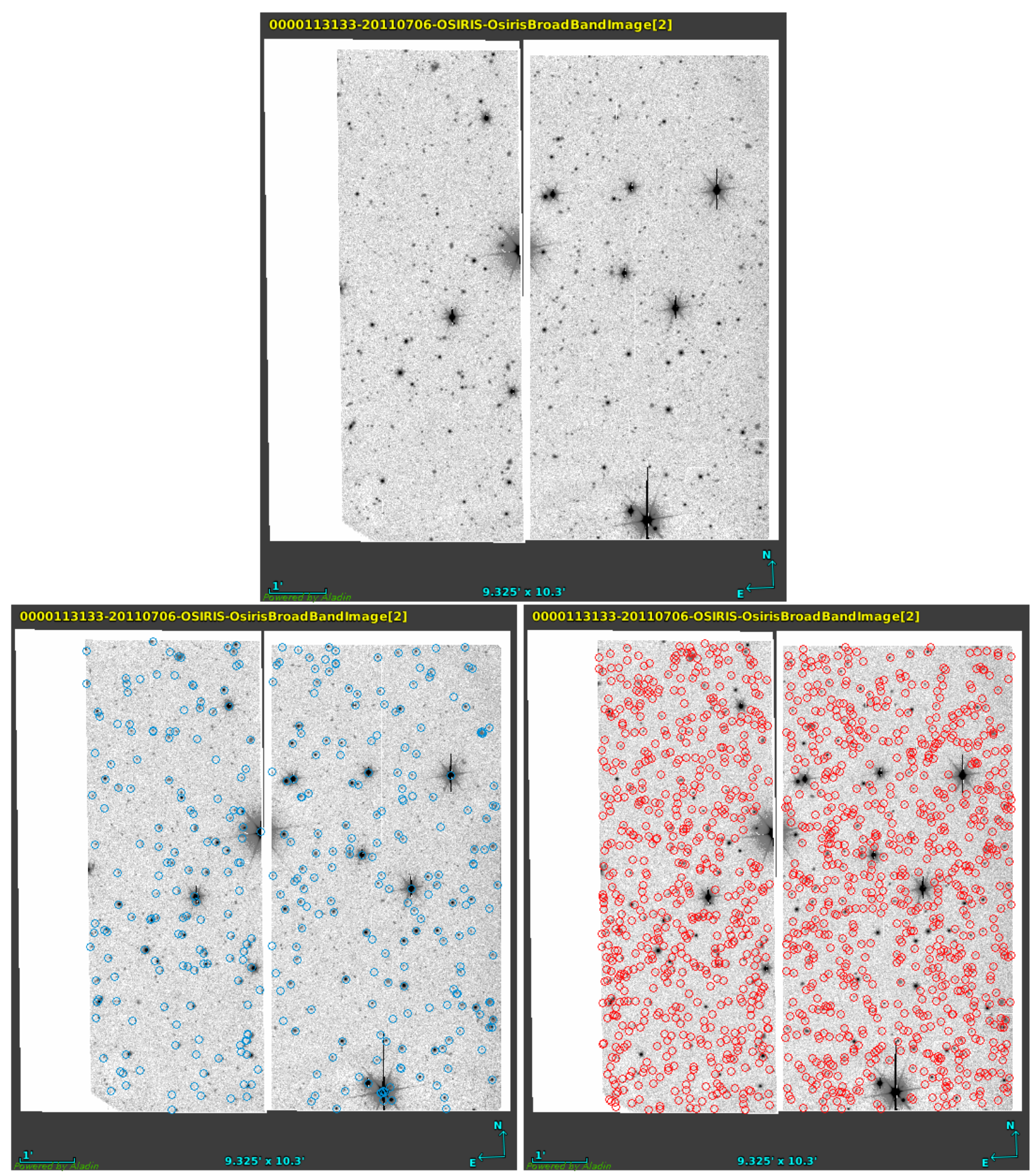

Figure 10. Example of an OSIRIS image centered at 17:06:59.64 +58:46:37.5 (top panel) overlayed with PS1 sources (bottom left panel, blue open circles) and OSIRIS sources (bottom right panel, red open circles).

of sources observed more than five times. The relation between their standard deviations and magnitudes is shown in Figure 16. We also show in this figure the averaged photometric errors of Figure 11. The scatter in the standard deviation of magnitudes increases towards fainter magnitudes in both, PSF and AUTO calibrated magnitudes. Also AUTO photometry shows slightly higher dispersion and error bars than PSF photometry. Since SExTRACTOR uses flexible elliptical apertures for extracting AUTO photometry, this effect can be attributed to the possibly different configurations applied to the same source in different images and epochs that lead to different flux integrations. In general, the variations of magnitudes of repeated sources is of the order of or lower than the mean accuracy of the catalogue within the 

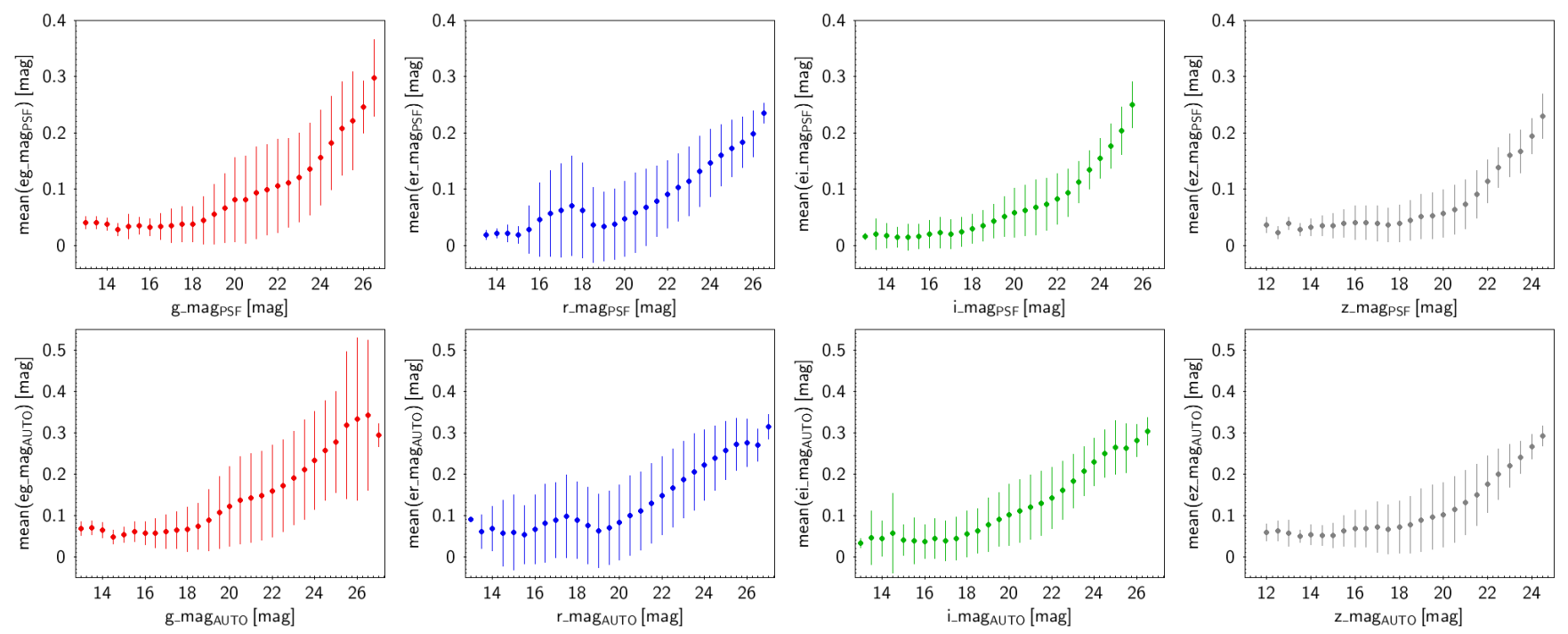

Figure 11. Photometric errors versus magnitudes for MAG_PSF (top) and MAG_AUTO (bottom) in the four bands. Bullets are the average values of the magnitude errors in bin sizes of $0.5 \mathrm{mag}$ and the error bars show the standard deviation.
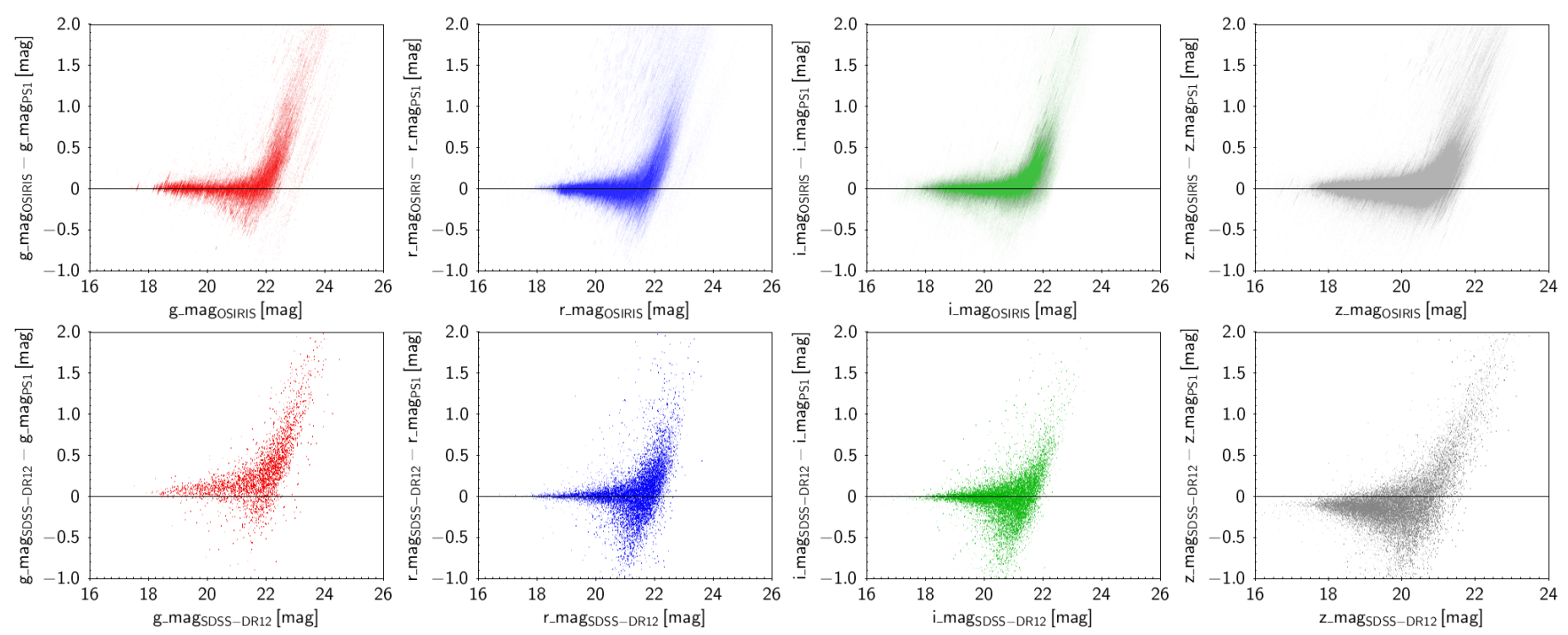

Figure 12. Comparison, for each filter, of PSF PS1 magnitudes with PSF OSIRIS (top panel) and of PSF PS1 magnitudes with PSF SDSS DR12 (bottom panel).

errorbars at each interval of magnitudes with the exception of the bright end of both, PSF and AUTO photometry, in the $g$ and $z$ bands. Also the faintest interval of magnitudes in the PSF panels in all bands and the AUTO photometry in the $i$ band show this behaviour, explained by the lower number of sources in this regime.

While this exercise is a good approach to assess the homogeneity and quality of the entire dataset, it can also be misleading since the tails of the distributions are generally caused by faint sources with low signal-to-noise ratio and a small number of artifacts that passed previous tests. Figure 17 shows, for each filter, the normalized cumulative histogram of the signal-to-noise ratio of sources with PSF magnitude standard deviations larger than the mean value plus $1 \sigma$ (this is, typically larger than $0.2 \mathrm{mag}$ ). Per filter, be- tween 75 and $85 \%$ of these problematic sources have SNR_WIN under 25 .

\subsubsection{Variability}

Real variable objects can also contribute to the tail of the distribution. We therefore looked for PSF magnitude variations according to the following equation:

$S=m a g_{\min }+3 * e \_m a g_{\min }-\left(\operatorname{mag} g_{\max }-3 * e \_\operatorname{mag} \max \right)$

where $m a g_{\min }, \operatorname{mag}_{\max }, e \_m a g_{\min }$ and $e \_m a g_{\max }$ are the lower and higher magnitudes measured for the same source and their associated errors. If $S$ is lower than zero, then the magnitude deviation of the source is outside the reach of three times the errorbars and cannot be explained by 


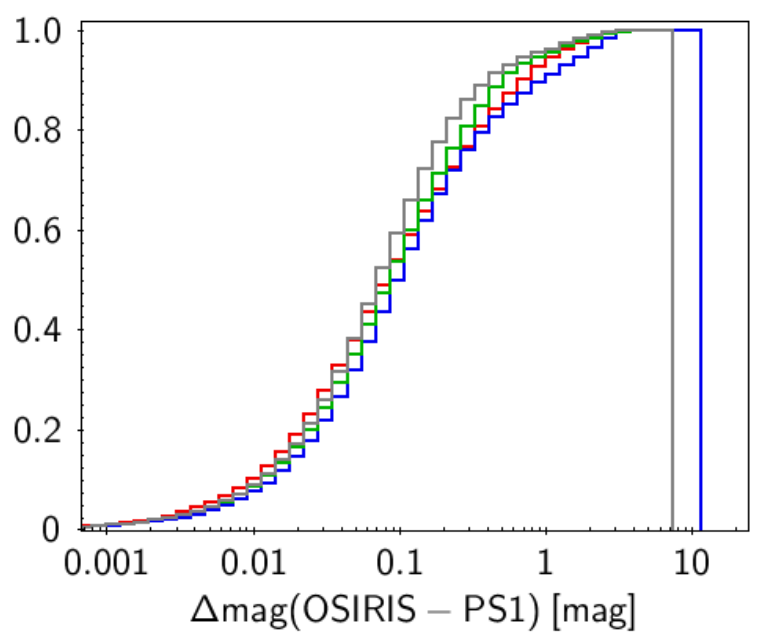

Figure 13. Normalized cumulative distribution of PSF absolute magnitude differences between OSIRIS and PS1 in logarithmic scale in the $g$ (red), $r$ (blue), $i$ (green) and $z$ (gray) bands.

the photometric errors. Of the 731685 sources that have been detected more than once in the same filter we found that, between 10 and $13 \%$, fulfill this criterion, depending on the filter. Figure 18 shows the PSF magnitude distributions of these sources in the four bands. They peak well over the $20.5-21.5 \mathrm{mag}$ limits stated for precise photometry and therefore, these magnitude variations could be related in some cases to the lower quality photometry of faint sources rather than to real photometric variation. Nonetheless, 33310 out of the 731685 repeated sources $(4.5 \%)$ are brighter than those magnitude limits $(21.5 \mathrm{mag}$ in $g$ and $r$, $21.0 \mathrm{mag}$ in $i$ and $20.5 \mathrm{mag}$ in $z$ ) and could actually be real photometric variable sources. Of them, only 16 have already been identified as variable in the literature. A proper analysis of the true nature of the variability detected in these sources is beyond the scope of this paper.

We also represent in Figure 19 the distributions of the SNR_WIN, elongation and ellipticity of the sources that show $S$ values smaller and greater than zero for comparison and in order to evaluate any drift of these parameters favouring variability. Although sources with $S>0$ register a mean signal-to-noise ratio slightly higher compared to the mean value of sources with $S<0$ ( 85 vs. 64 ), the peak in both signal-to-noise ratio distributions is similar. The behaviour of morphometric parameters is comparable in both samples as well. Hence, we do not see any dependence of the photometric variability with lower signal-to-noise ratio or flattening of the sources.

On the other hand, we also investigated if known variable sources do also show variability in our catalogue. To do so, we cross-matched our catalogue with Gaia DR2 and the AAVSO International Variable Star Index VSX (Watson et al. 2006). Only 73 different sources in our catalogue are tagged as variables in these catalogues. We looked in the SIMBAD astronomical database (Wenger et al. 2000) and identified another 319 sources classified as variables or suspected of variability. We show in Figure 20 the PSF standard deviation of magnitudes for each of these sources with respect to their PSF magnitudes in the corresponding filter. The majority of these sources do not present significant variations except perhaps the five sources labeled in the plot with standard deviations over $0.2 \mathrm{mag}$ and larger than their magnitude errors. Sources \#1 and \#2 correspond to the same source (NSV 13246) observed in the $g$ and $r$ bands, and classified in Gaia DR2 and SIMBAD as Fundamentalmode RR Lyrae star; source \#3 (SN 2011by) is a SN Ia in the AAVSO International Variable Star Index VSX catalogue; source \#4 (CRTS J154326.0-212800) is classified in Gaia DR2 and SIMBAD as a fundamental-mode RR Lyrae star; and source \#5 (2MASS J20325377+4115134) is found in SIMBAD as an eclipsing binary candidate, variable and $\mathrm{X}$ ray emitting source. Sources \#1 and \#2 have been detected within 0.83 years four and two times in our catalogue, respectively. Sources \#3, \#4 and \#5 have been detected twice in 56 days, six times within 10 days and six times within 18 days, respectively. Due to the time span of the observations, these magnitude variations could probably be associated to real photometric variations in all cases. Unfortunately, the cadence in the observations might not be suitable for variability detection in the catalogue.

\section{SCIENTIFIC EXPLOITATION}

To illustrate the science capabilities of the catalogue, we defined two cases to prove the goodness of the photometry (identification of cool stars) and the astrometry (identification of asteroids) provided in the catalogue.

\subsection{Looking for cool dwarfs}

Cool dwarfs of M spectral type constitute around the $40 \%$ of the stellar mass in the Galaxy (Gould et al. 1996; Bochanski et al. 2010). Their ubiquity and lifetimes that exceed the current age of the Universe (Baraffe et al. 1998; Henry et al. 2006) makes them excellent targets to broadly study the formation and evolution processes at the bottom of the main-sequence.

To identify cool dwarfs in the OSIRIS catalogue, we used the $i$ and $z$ bands and selected all sources with $i-$ $z>0.38 \mathrm{mag}$, which would correspond to spectral types later than M0 according to West et al. (2008). We then cross-matched the selected sources with Gaia DR2 within 1.5 arcsec and kept all sources with relative errors in parallax and proper motion below $20 \%$. Later we removed all sources with proper motions $\mu<30$ mas/yr as in Solano et al. (2019) to avoid contamination of giants and subgiants in the sample. This way, we ended up with 52 cool dwarf candidates, for which we derived effective temperatures in order to confirm their cool nature. To do so, we used VOSA (Bayo et al. 2008) which allowed us to gather photometry from the DENIS (Epchtein et al. 1999; Fouqué et al. 2000), 2MASS (Skrutskie et al. 2006), UKIDSS (Hewett et al. 2006; Casali et al. 2007; Lawrence et al. 2007b; Hodgkin et al. 2009), IPHAS (González-Solares et al. 2008; Barentsen et al. 2014), WISE (Wright et al. 2010b), SDSS DR9 (Ahn et al. 2012), Pan-STARRS DR1 (Chambers et al. 2016; Flewelling et al. 2016; Magnier et al. 2016a,b,c; Tonry et al. 2012; Waters et al. 2016) and Gaia DR2 catalogues. This information was used together the photometric points in the griz bands from our OSIRIS catalogue making use of the SVO Filter Profile Service to build the corresponding Spectral Energy 

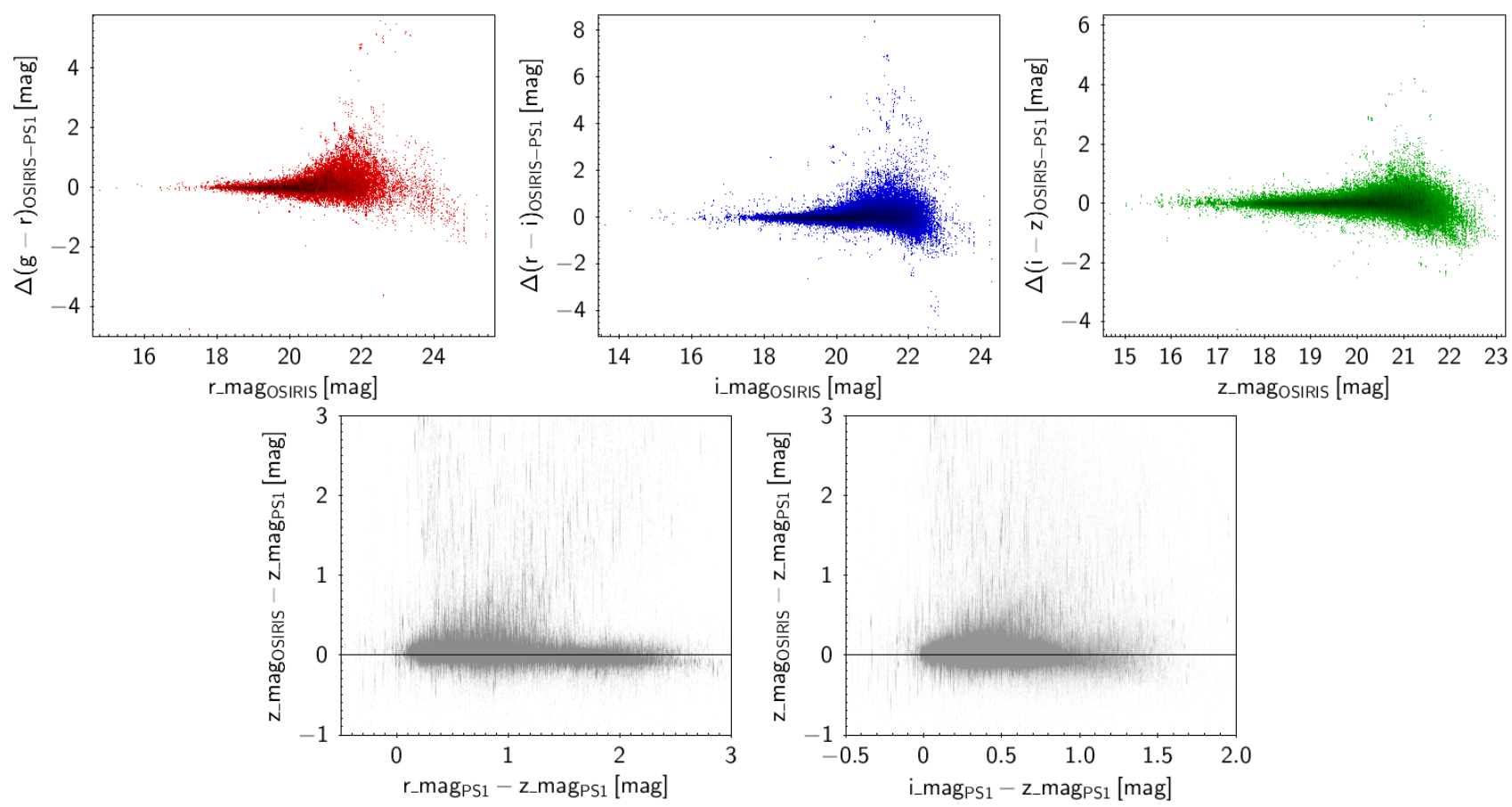

Figure 14. Top panel: Dependence of the difference of colours (OSIRIS - PS1) with OSIRIS PSF magnitudes. Bottom panel: Dependence of the difference of magnitudes in the $\mathrm{z}$ band with PS1 $r-z$ (left) and $i-z$ (right) colours.
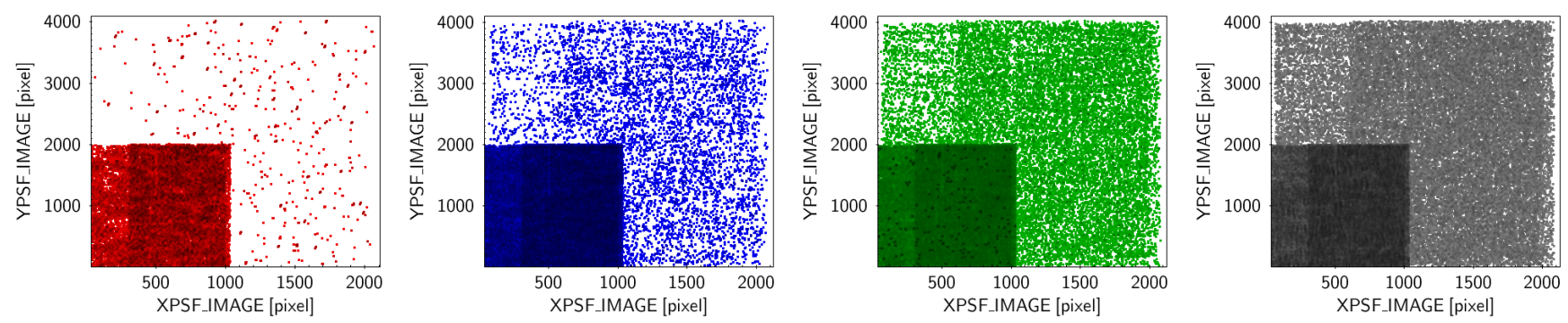

Figure 15. CCD $x y$ position of sources with PS1 photometry and fainter PSF magnitudes than 21.5 mag in $g$ (red panel) and $r$ (blue panel), $21.0 \mathrm{mag}$ in $i$ (green panel), and $20.5 \mathrm{mag}$ in $z$ (gray panel).

Distributions (SEDs). We applied the BT-Settl collection of theoretical models (Allard et al. 2012) with solar metallicity, $\log g$ between 4.5 and 6.0 and $T_{\text {eff }}$ between 1000 and $5000 \mathrm{~K}$. We left extinction $A_{V}$ as a free parameter varying from 0 to $1 \mathrm{mag}$ (assuming that extinction could be up to $1 \mathrm{mag}$ at $1 \mathrm{kpc}$ ) since it can strongly modify the shape of the SED and, therefore, the parameters determination. VOSA fittings were visually inspected to confirm that OSIRIS photometry does not deviate from the SED.

The lack of good photometry prevented us from performing a reliable fit for three out of the 52 candidates. One of these three turned out to be a non-catalogued resolved physical binary with a late-type primary separated $1.24 \operatorname{arcsec}$ at $195 \mathrm{pc}(241.7 \mathrm{AU})$. Near a hundred binaries with late $\mathrm{M} / \mathrm{L}$ dwarf primaries have been identified up to date (Bouy et al. 2003; Close et al. 2003; Dupuy \& Liu 2017; Bardalez Gagliuffi et al. 2015). 2MASS, Gaia DR2 and GTC OSIRIS are able to resolve the pair and we can therefore confirm common proper motion. We could estimate the spectral type of the components from the Gaia DR2 $G-R P$ colours and the updated version of Table 5 in Pecaut \& Mamajek $(2013)^{7}$. The primary (GTC_OSIRIS_BBI_DR1_J203146.23+411437.0) would be an $\mathrm{M} 9.5 \mathrm{~V}$ with $G-R P=1.627 \mathrm{mag}$. For the secondary (GTC_OSIRIS_BBI_DR1_J203146.19+411437.6), there is no RP photometry. From the $\Delta G=1.0 \mathrm{mag}$ of the system, we estimate the secondary to be an L1-L2 dwarf. Another one of these three sources (GTC_OSIRIS_BBI_DR1_J181631.68+691152.9) has a close bright companion in our OSIRIS images at 2.3 arcsec that saturates (and is therefore not in the catalogue) and that is not resolved by Gaia DR2, PS1, 2MASS or WISE. The combined photometry of the two sources in these catalogues prevent us from obtaining the SED of our cool dwarf candidate. We can not suggest nor discard physical binding due to the lack of available information.

\footnotetext{
7 http://www.pas.rochester.edu/ ${ }^{\sim}$ emamajek/EEM_dwarf_ UBVIJHK_colors_Teff.txt
} 

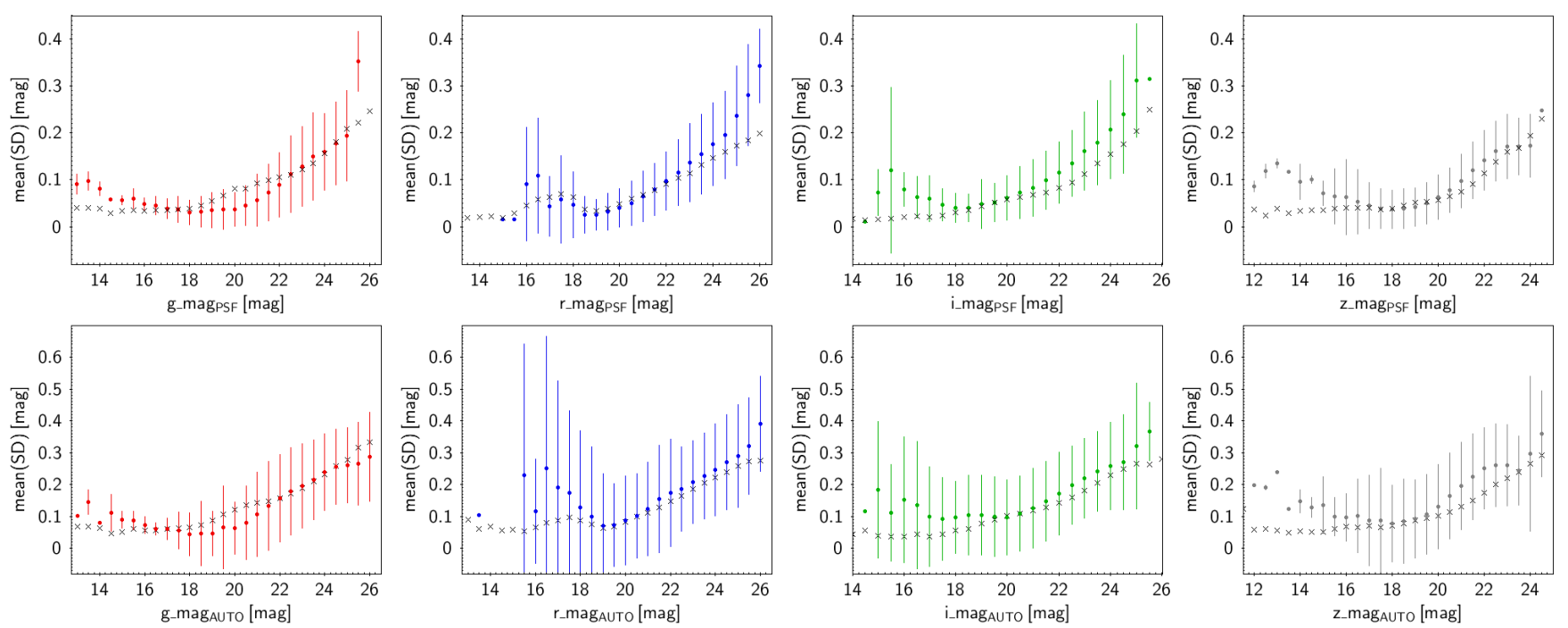

Figure 16. Photometric repeatability as a function of magnitudes for sources observed more than five times for PSF (top) and AUTO (bottom) photometry. Bullets are the average values of the standard deviations of magnitudes of repeated sources in bin sizes of 0.5 mag and the error bars show their standard deviations. Mean values of the average photometric errors shown in Figure 11 are displayed as black crosses.

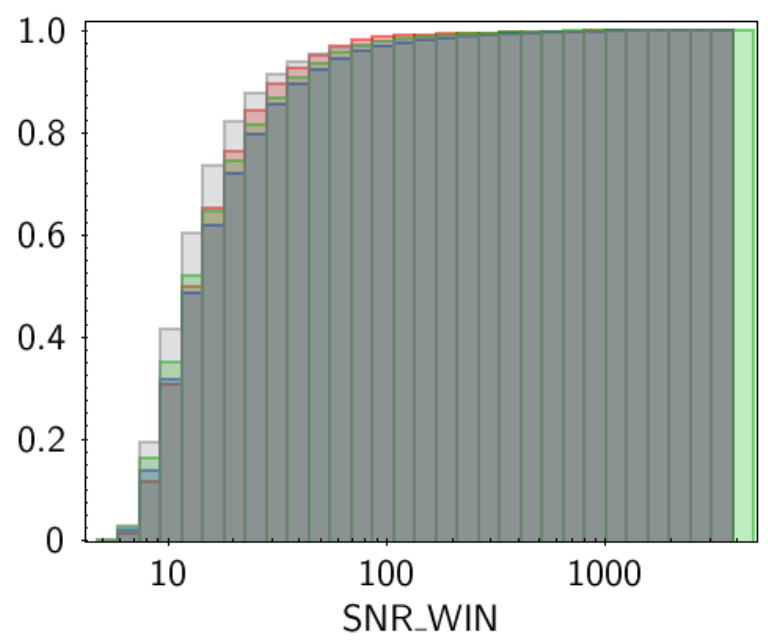

Figure 17. Normalized cumulative distributions of the signal-tonoise ratios of sources observed more than five times and with large standard deviation of PSF magnitudes. The $g, r, i$ and $z$ filters are represented in red, blue, green and gray, respectively.

In addition, we obtained effective temperatures for 49 sources ranging from 2400 to $3700 \mathrm{~K}$ with an uncertainty of $50 \mathrm{~K}$. Their positions shown in the colour-magnitude diagram of Figure 21 built with Gaia DR2 sources also agree with being sources later than M0. Their distances range between 75.9 and $854.6 \mathrm{pc}$. The coolest dwarf in the sample lies in the $\mathrm{M} / \mathrm{L}$ transition region at $85.9 \mathrm{pc}$. With an effective temperature of $2400 \mathrm{~K}$ we estimate it's spectral type to be an M9.0-M9.5 dwarf. Again, using the Gaia $G-R P$ colour and the updated version of Table 5 in Pecaut \& Mamajek (2013), we infer a spectral type between M9.5 and L0.

Among the 49 cool sources, we found another non previously reported close binary separated 3.84 arcsec (1940.4 AU). We confirm common proper motion from

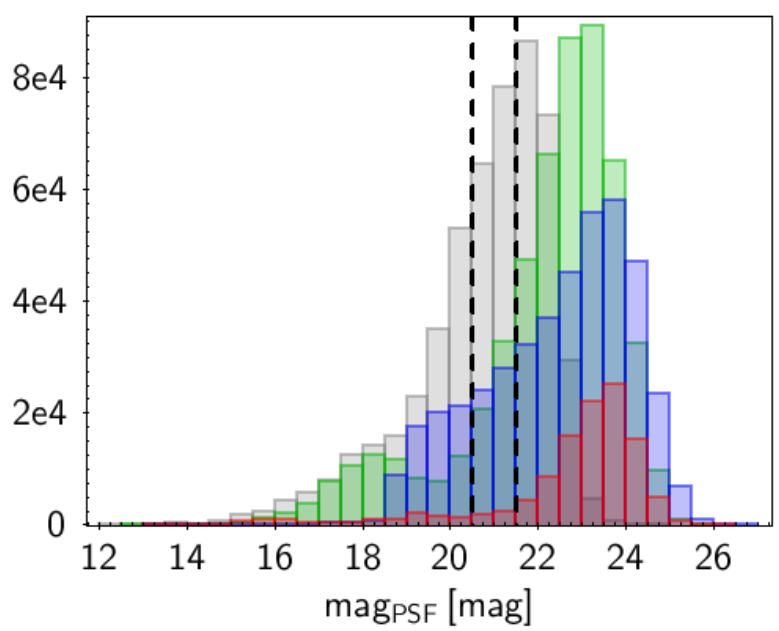

Figure 18. PSF magnitude distributions of sources with $S<0$ in the $g$ (red), $r$ (blue), $i$ (green) and $z$ (gray) bands. Vertical black dashed lines indicate the 20.5-21.5 mag limits of precise photometry.

the detection of both components by UKIDSS, PanSTARRS DR1 and Gaia DR2. The pair is located at $505.3 \mathrm{pc}$. In this work, we identified the secondary source of the system (GTC_OSIRIS_BBI_DR1_J203324.41+410751.7) and obtained an effective temperature of $3300 \mathrm{~K}$. For the primary (not included in the OSIRIS catalogue because of saturation in all images), we determined an effective temperature of $4200 \mathrm{~K}$ using VOSA. From the Gaia DR2 $G-R P$ colours and the updated version of Table 5 in Pecaut \& Mamajek (2013), we estimated the spectral type of the components to be a K7 and an M4.0-M4.5. Spectral types are in agreement with the derived temperatures.

Only three out of the 49 sources were found in SIMBAD and none of them have been reported as cool type stars. As a 

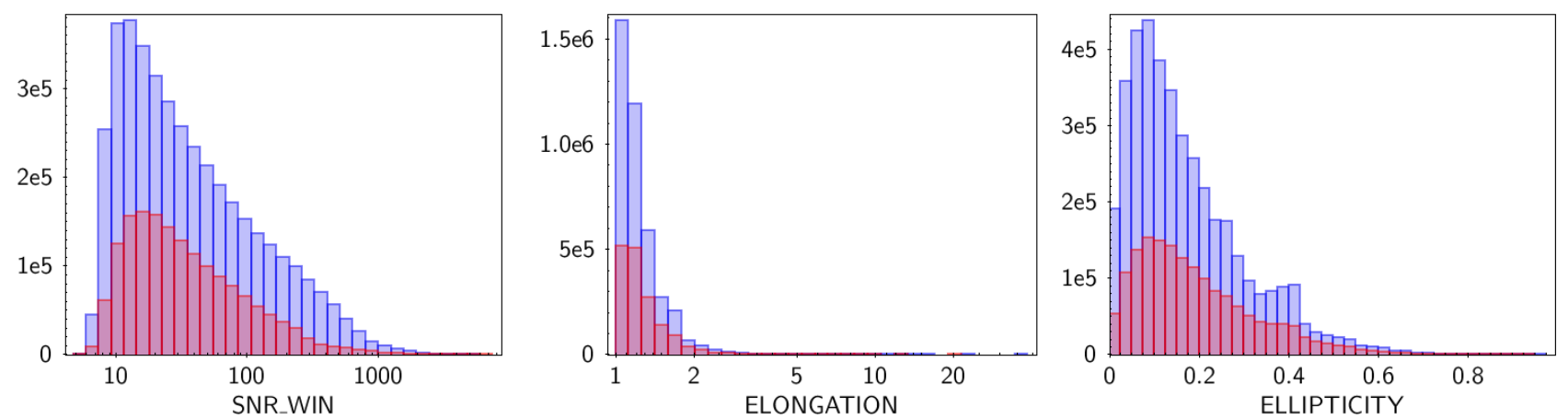

Figure 19. Distribution of the SNR (left), elongation (middle) and ellipticity (right) of repeated sources in the catalogue with $S<0$ (red) and with $S>0$ (blue).

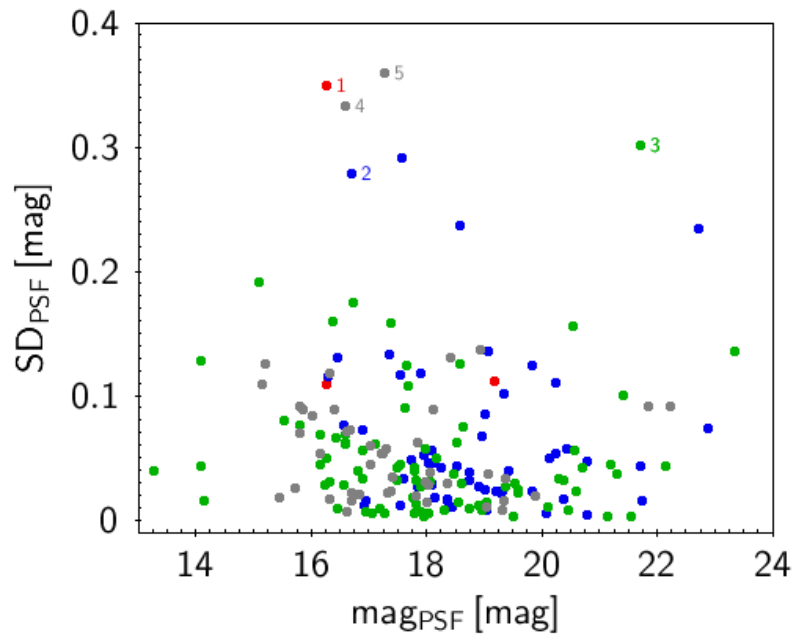

Figure 20. Standard deviation of PSF magnitudes of known variable sources. Red, blue, green and gray filled circles stand for $g$, $r, i$ and $z$ bands respectively.

curiosity, 36 out of the 49 sources cover less than one square degree of the sky in the region of the Cygnus OB association, although they are found at less than half its distance. This is a region of high scientific interest and may have therefore been often observed in several bands. This would explain the large fraction of cool objects found in this region.

Table C1 lists the OSIRIS and Gaia DR2 identifiers and effective temperatures of the 49 dwarf candidates and the identifiers of the late-type binary components.

\subsection{Identification of asteroids}

We applied the ssos $^{8}$ pipeline (Mahlke et al. 2019) to detect and identify Solar System Objects (SSOs) serendipitously observed in the OSIRIS images. The pipeline detects both known and unknown SSOs primarily based on their linear apparent motion in subsequent exposures. Source detection and association are performed by SEXTRACTOR and SCAMP respectively, while the separation of SSOs from other sources in the image catalogues is performed by a chain

\footnotetext{
8 https://pypi.org/project/ssos/
}

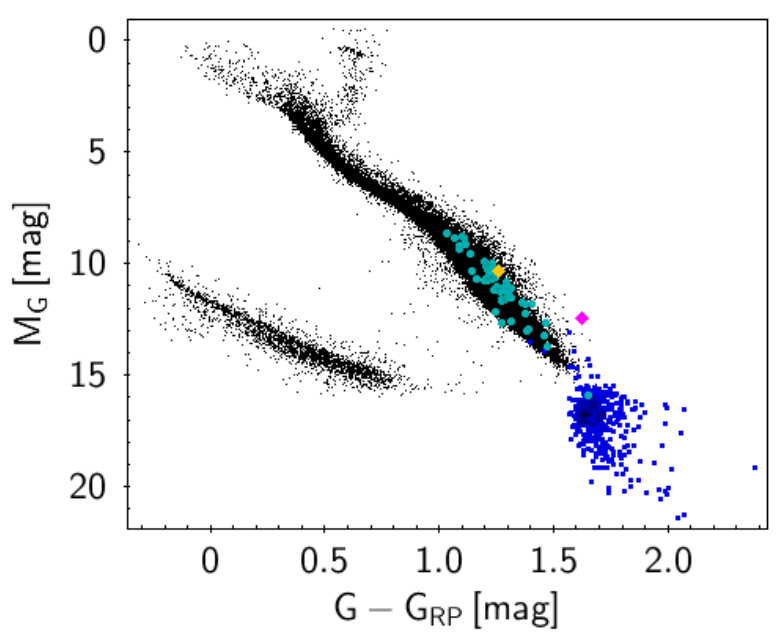

Figure 21. Colour-magnitude diagram using Gaia DR2 sources with parallaxes larger than 10 mas (black dots). Light blue filled circles represent our 48 single dwarf candidates later than M0, yellow filled diamond represents the $\mathrm{M}$ type secondary belonging to the $\mathrm{K}+\mathrm{M}$ close binary and the magenta filled diamond stands for the $\mathrm{M}+\mathrm{L}$ close binary system. L and $\mathrm{T}$ dwarfs with Gaia counterparts identified in Smart et al. (2017) are displayed with dark blue dots.

of user-configurable filter algorithms. A more detailed discussion of the pipeline and its application to the OSIRIS images can be found in Mahlke et al. (2019).

To apply the pipeline, the images were grouped by observation night and overlapping field-of-views. Of the full sample of images in the DR1, we built 420 groups made up of 6982 images. The remaining images had to be discarded as there were fewer than 4 exposures in the respective visits, a requirement for a reliable detection of SSOs.

Applying the ssos pipeline to these 420 groups revealed 204 unique SSOs present in a total of 2828 images. 63 objects could be identified as known SSOs using the IMCCE's SkyBoT service, which computes the ephemerides of SSOs within a given field-of-view and observation epoch. The returned computed ephemerides were cross-matched within a radius of 40 arcsec with the positions of the recovered SSOs. Table 4 lists the classes of the 63 identified objects. The majority are Main-Belt (MB) asteroids. Four comets were retrieved as well, however, they were the targets of the respective observations. Figure 22 depicts four detections of 


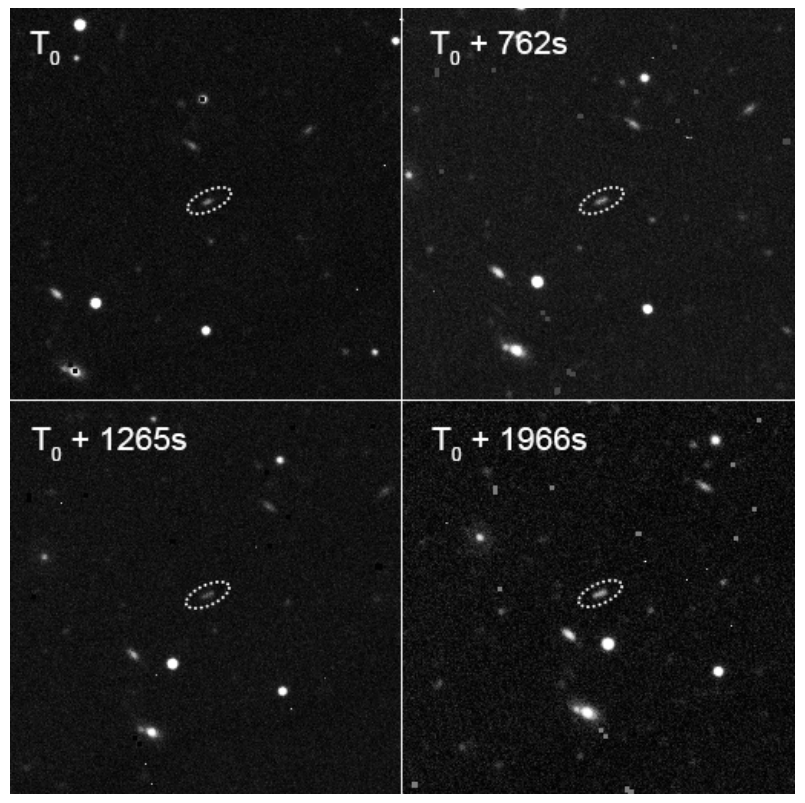

Figure 22. Four observations of (355891) 2008 WE46 recovered from the DR1 images. The main-belt asteroid was serendipitously observed and recovered using the ssos pipeline. Its position is marked by the dotted white ellipse in the center of each frame. The text indicates the time difference between the frames in seconds.

(355891) 2008 WE46 to illustrate the serendipitous observations.

The remaining 141 SSOs are either unknown or had a discrepancy between predicted and observed position larger than 40 arcsec, meaning that their observation will greatly improve the accuracy of their orbit. The astrometric and photometric properties of all 2828 SSO detections have been reported to the Minor Planet Centre 9 (MPC). Of the 1002 observations of known SSOs, 872 were ingested into the MPC database ${ }^{10}$. The remaining 130 observations have not been published due to unknown reasons. The 1826 observations of unknown SSOs consist of single-night observations only. Therefore, they will not receive temporary designations, however, they are ingested into the MPC database and might eventually be associated to a newly discovered object.

Unfortunately, the acquired photometry did not allow for spectrophotometric classification of the SSOs as the observations typically spanned more than 1 hour, rendering the determination of colours unreliable. Moreover, the temporal baseline was not long enough to estimate rotation periods from the light curve analysis. The observations will still be useful in combination with other data, however, to determine e.g. the SSO phase function parameters.

\footnotetext{
9 https://www.minorplanetcenter.net/

10 https://minorplanetcenter.net/iau/ECS/MPCArchive/

2018/MPS_20181118.pdf, observatory code Z18
}

Table 4. Distribution of previously known SSOs detected in the DR1 images over SSO classes. The 4 comets were retrieved from targeted observations. MB stands for Main-Belt.

\begin{tabular}{lllll}
\hline \hline Comet & Inner MB & Middle MB & Outer MB & Trojan \\
\hline 4 & 21 & 17 & 20 & 1 \\
\hline
\end{tabular}

\section{DATA ACCESS}

The photometrically and astrometrically corrected OSIRIS broadband images as well as the associated catalogue are available to the community through the GTC Archive Por$\mathrm{tal}^{11}$ or the associated Virtual Observatory services (SIAP for images and ConeSearch for the catalogue). The GTC archive is maintained by Centro de Astrobiologia (INTACSIC) in the framework of the Spanish Virtual Observatory $^{12}$. The results provided by the portal or the VO services can be sent using the SAMP protocol to other VO tools for its further visualization and/or analysis.

In order to help the astronomical community on using the detection and source catalogues built from the OSIRIS broadband images, we have developed an archive system that can be accessed from a webpage ${ }^{13}$ or through a Virtual Observatory ConeSearch ${ }^{14}$.

The archive system implements a very simple search interface (see Fig 23) that permits queries by position or PSF magnitude interval in both, detection and source catalogues, and also by colour range only in the source catalogue. The system implements aswell a link to the images in GTC Public Archive.

The result of the query is a HTML table with all the sources found in the archive fulfilling the search criteria up to a limit of 10000 lines. The result can also be downloaded as a VOTable or a CSV file. Detailed information on the output fields can be obtained placing the mouse over the question mark ("?") located close to the name of the column. The archive also implements the SAMP ${ }^{15}$ (Simple Application Messaging) Virtual Observatory protocol. SAMP allows Virtual Observatory applications to communicate with each other in a seamless and transparent manner for the user. This way, the results of a query can be easily transferred to other VO applications, such as, for instance, TOPCAT.

\section{CONCLUSIONS AND FUTURE WORK}

We have presented the database of processed and scientific grade broadband images and the associated catalogue obtained with the GTC OSIRIS instrument from April 2009 to January 2014. In this first release, the database includes 6788 images in the Sloan griz bands with exposure times

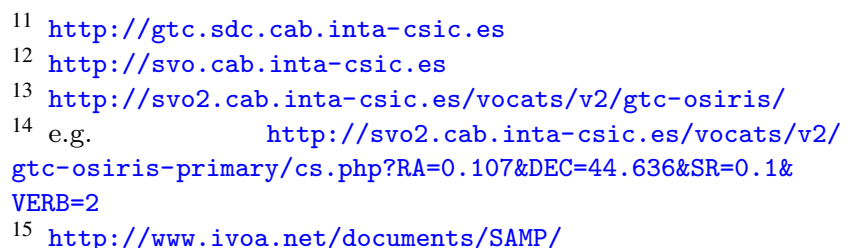




\section{GTC OSIRIS Broad Band First Data Release: Detection catalogue}

\section{Home Data retrieval News Documentation $/$ Coverage Map Credits Help-desk}

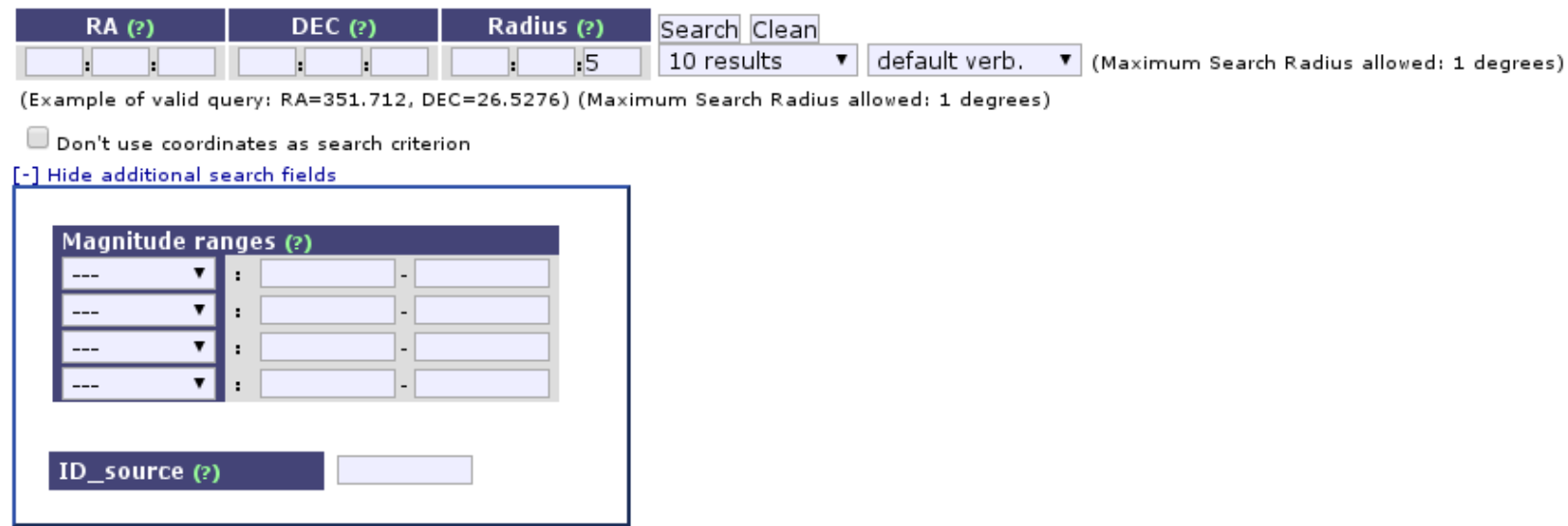

\section{GTC OSIRIS Broad Band First Data Release: Source catalogue}

\section{Home Data retrieval News Documentation Coverage Map Credits Help-desk}

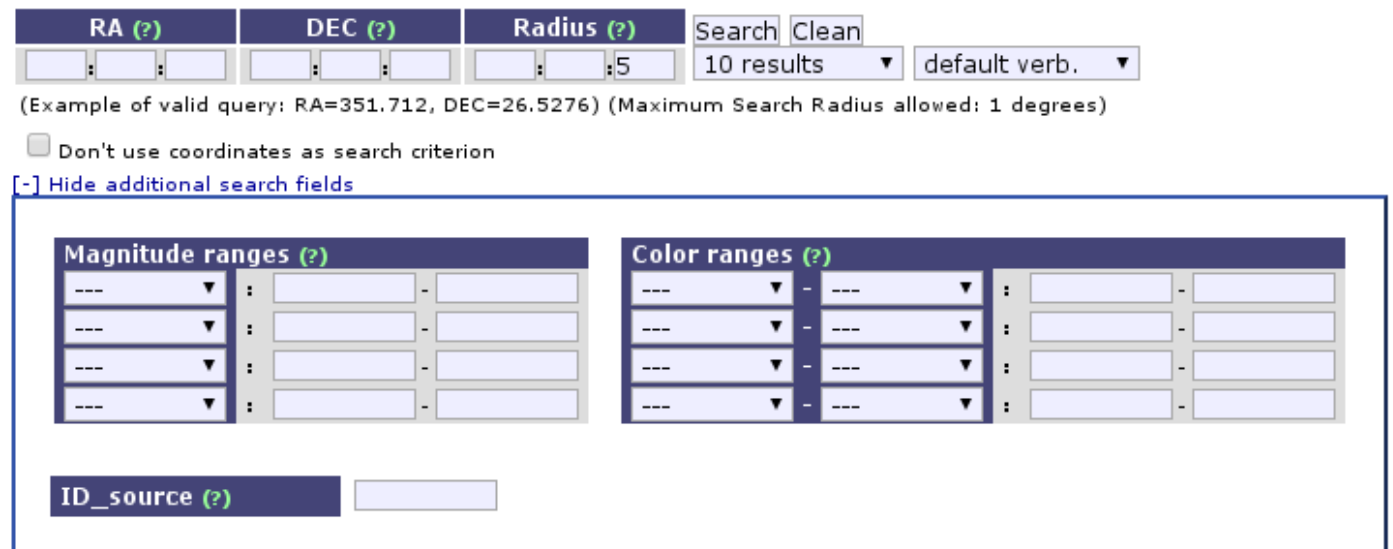

Figure 23. Screenshots of the archive search interfaces that permit simple queries to the detection (top) and source (bottom) catalogues.

ranging from 0.5 to $900 \mathrm{~s}$, and 6226520 entries in the catalogue corresponding to 633559 astronomical sources. The catalogue is astrometrically and photometrically calibrated in standard PSF and automatic (Kron equivalent) photometry using 2MASS, SDSS DR10 or USNO-B1 for the astrometry and Pan-STARRS DR1 for the photometry, allowing a broad range of scientific activities.

Relative astrometric residuals typically are within 30 mas and typical positional uncertainty is of 0.12 arcsec. Only well correlated sources between instrumental and PanSTARRS DR1 magnitudes were used for photometric cali- bration, providing a precision of 0.034-0.056 mag, depending on the filter.

The mean photometric accuracy of the whole catalogue is better than $0.09 \mathrm{mag}$ and $0.15 \mathrm{mag}$ in PSF and AUTO photometry, respectively. Besides, a comparison with PANSTARRS DR1 good quality photometry presents magnitude differences under $0.18 \mathrm{mag}$ in all bands. Additional tests did not reveal any colour term in the photometric calibration nor dependence with the binning mode of observations nor the pixel position in the CCD.

Saturation and typical magnitude limits in the cata- 
logue in each filter are 13.1-24.6 mag in $g, 13.6-24.5 \mathrm{mag}$ in $r, 13.1-23.9 \mathrm{mag}$ in $i$, and $12.0-22.7 \mathrm{mag}$ in $z$, respectively.

We present two science cases aiming to prove the scientific capabilities of the catalogue. In the first case, we looked for cool dwarfs in the catalogue using a photometric criterium in the optical and Gaia DR2 astrometry. We identified 49 dwarfs of spectral types later than M0 according to their positions in the colour-magnitude diagram and their effective temperatures, with distances between 75.9 and $854.6 \mathrm{pc}$, which need spectroscopic confirmation. Among them, we identified a new resolved binary system separated $3.84 \operatorname{arcsec}(1940.4 \mathrm{AU})$ composed by a late K primary and a mid type M secondary. Additionally, we identified another new resolved binary system separated $1.24 \operatorname{arcsec}(247.7 \mathrm{AU})$ with estimated spectral types M9.5 + L1/L2 dwarfs. We propose them for follow up and spectroscopic confirmation. In the second case, we looked for Solar System Objects from their apparent linear motion and identified 59 known asteroids and four known comets plus 141 unknown objects or objects with poor accuracy in their orbital elements. For them, we provide 2828 detections that have been reported to the Minor Planet Center Database.

The catalogue includes celestial coordinates, sources and detections IDs, calibrated PSF and Kron photometry in the griz Sloan bands, various morphometric measurements useful to assess the nature of the sources (point-like or extended), although we provide a point-like/extended classification and a point-like coefficient. In addition, it contains two quality flags associated to the photometric coverage of the calibration using PAN-STARRS DR1 catalogue. Instrumental magnitudes of MODEL and aperture photometry, and epoch of observation are also included in the catalogue. The user can access the raw and processed images through an url given in the catalogue.

New releases of the catalogue including the new public broadband images will be delivered over the life of the instrument. Also, a number of improvements and enhancements are planned for these future releases. One of the most important ones will be the building of stacked images to develop a much deeper catalogue. We also plan to improve the absolute astrometry of the catalogue by linking it to the Gaia DR2 catalogue. New versions of the Pan-Starrs will also be used for the photometric calibration.

Like in any other photometric survey, the complete absence of errors and problems in the catalogue cannot be guaranteed, in particular for detections close to the limiting magnitude. In these cases, users are strongly encouraged to download and check the associated images to assess the reliability of a given catalogue measurement. The News section of the catalogue website will contain a list of Frequently Asked Questions as well as a description of caveats that may arise with the scientific exploitation of the catalogue.

\section{ACKNOWLEDGMENTS}

H. Bouy acknowledges funding from the European Research Council (ERC) under the European Union's Horizon 2020 research and innovation programme (grant agreement No 682903 , P.I. H. Bouy), and from the French State in the framework of the "Investments for the future" Program, IdEx Bordeaux, reference ANR-10-IDEX-03-02. This re- search has been financed by ASTERICS, a project supported by the European Commission Framework Programme Horizon 2020 Research and Innovation action under grant agreement n. 653477. This research has been partially funded by the Spanish State Research Agency (AEI) Project No. ESP2017-87676-C5-1-R and No. MDM-2017-0737 Unidad de Excelencia "María de Maeztu"- Centro de Astrobiología (CSIC-INTA). This publication makes use of VOSA, developed under the Spanish Virtual Observatory project supported by the Spanish MINECO through grant AyA201784089. This research has made use of the SVO Filter Profile Service (http://svo2.cab.inta-csic.es/theory/fps/) supported from the Spanish MINECO through grant AYA201784089. This research made use of the cross-match service, the SIMBAD database (Wenger et al. 2000), VizieR catalogue access tool (Ochsenbein et al. 2000), "Aladin sky atlas" (Bonnarel et al. 2000; Boch \& Fernique 2014) provided by CDS, Strasbourg, France. This research has also made use of the TOPCAT (Taylor 2005) and STILTS (Taylor 2006). MCC and FJE acknowledge financial support from the Tec2Space-CM project (P2018/NMT-4291). MM is funded by the European Space Agency under the research contract C4000122918.

\section{REFERENCES}

Ahn C. P., et al., 2012, ApJS, 203, 21

Ahn C. P., et al., 2014, ApJS, 211, 17

Allard F., Homeier D., Freytag B., 2012, Philosophical Transactions of the Royal Society of London Series A, 370, 2765

Baraffe I., Chabrier G., Allard F., Hauschildt P. H., 1998, A\&A, 337,403

Bardalez Gagliuffi D. C., Gelino C. R., Burgasser A. J., 2015, AJ, 150,163

Barentsen G., et al., 2014, MNRAS, 444, 3230

Bayo A., Rodrigo C., Barrado Y Navascués D., Solano E., Gutiérrez R., Morales-Calderón M., Allard F., 2008, A\&A, 492, 277

Bertin E., 2006, in Gabriel C., Arviset C., Ponz D., Enrique S., eds, Astronomical Society of the Pacific Conference Series Vol. 351, Astronomical Data Analysis Software and Systems XV. p. 112

Bertin E., 2013, PSFEx: Point Spread Function Extractor, Astrophysics Source Code Library (ascl:1301.001)

Bertin E., Arnouts S., 1996, Astronomy \& Astrophysics Supplement, 117, 393

Bertin E., Mellier Y., Radovich M., Missonnier G., Didelon P., Morin B., 2002, in Bohlender D. A., Durand D., Handley T. H., eds, Astronomical Society of the Pacific Conference Series Vol. 281, Astronomical Data Analysis Software and Systems XI. p. 228

Boch T., Fernique P., 2014, in Manset N., Forshay P., eds, Astronomical Society of the Pacific Conference Series Vol. 485, Astronomical Data Analysis Software and Systems XXIII. p. 277

Bochanski J. J., Hawley S. L., Covey K. R., West A. A., Reid I. N., Golimowski D. A., Ivezić Ž., 2010, AJ, 139, 2679

Bonnarel F., et al., 2000, A\&AS, 143, 33

Bouy H., Brandner W., Martín E. L., Delfosse X., Allard F., Basri G., 2003, AJ, 126, 1526

Bouy H., Bertin E., Moraux E., Cuillandre J.-C., Bouvier J., Barrado D., Solano E., Bayo A., 2013, Astronomy \& Astrophysics, 554, A101

Casali M., et al., 2007, A\&A, 467, 777

Chambers K. C., et al., 2016, preprint, (arXiv:1612.05560)

Close L. M., Siegler N., Freed M., Biller B., 2003, ApJ, 587, 407

Cutri R. M., et al., 2003, VizieR Online Data Catalog, 2246, 0 
Dupuy T. J., Liu M. C., 2017, ApJS, 231, 15

Epchtein N., et al., 1999, A\&A, 349, 236

Farrow D. J., et al., 2014, MNRAS, 437, 748

Flewelling H. A., et al., 2016, arXiv e-prints, p. arXiv:1612.05243

Fouqué P., et al., 2000, A\&AS, 141, 313

González-Solares E. A., et al., 2008, MNRAS, 388, 89

Gould A., Bahcall J. N., Flynn C., 1996, ApJ, 465, 759

Henry T. J., Jao W.-C., Subasavage J. P., Beaulieu T. D., Ianna P. A., Costa E., Méndez R. A., 2006, AJ, 132, 2360

Hewett P. C., Warren S. J., Leggett S. K., Hodgkin S. T., 2006, MNRAS, 367, 454

Hodgkin S. T., Irwin M. J., Hewett P. C., Warren S. J., 2009, MNRAS, 394, 675

Kaiser N., et al., 2010, The Pan-STARRS wide-field optical/NIR imaging survey, doi:10.1117/12.859188, https://doi.org/10. $1117 / 12.859188$

Lawrence A., et al., 2007a, MNRAS, 379, 1599

Lawrence A., et al., 2007b, MNRAS, 379, 1599

Magnier E. A., et al., 2016a, preprint, (arXiv:1612.05242)

Magnier E. A., et al., 2016b, arXiv e-prints, p. arXiv:1612.05240

Magnier E. A., et al., 2016c, arXiv e-prints, p. arXiv:1612.05244

Mahlke M., Solano E., Bouy H., Carry B., Verdoes Kleijn G. A., Bertin E., 2019, Astronomy and Computing, 28, 100289

Monet D. G., et al., 2003, AJ, 125, 984

Ochsenbein F., Bauer P., Marcout J., 2000, A\&AS, 143, 23

Paillassa M., Bertin E., Bouy H., 2019, arXiv e-prints, p. arXiv: 1907.08298

Pecaut M. J., Mamajek E. E., 2013, ApJS, 208, 9

Skrutskie M. F., et al., 2006, AJ, 131, 1163

Smart R. L., Marocco F., Caballero J. A., Jones H. R. A., Barrado D., Beamín J. C., Pinfield D. J., Sarro L. M., 2017, MNRAS, 469, 401

Solano E., et al., 2019, A\&A, 627, A29

Taylor M. B., 2005, in Shopbell P., Britton M., Ebert R., eds, Astronomical Society of the Pacific Conference Series Vol. 347, Astronomical Data Analysis Software and Systems XIV. p. 29

Taylor M. B., 2006, in Gabriel C., Arviset C., Ponz D., Enrique S., eds, Astronomical Society of the Pacific Conference Series Vol. 351, Astronomical Data Analysis Software and Systems XV. p. 666

Tonry J. L., et al., 2012, ApJ, 750, 99

Vandame B., 2002, in Starck J.-L., Murtagh F. D., eds, Society of Photo-Optical Instrumentation Engineers (SPIE) Conference Series Vol. 4847, Astronomical Data Analysis II. pp 123-134 (arXiv: astro-ph/0208230), doi:10.1117/12.460591

Waters C. Z., et al., 2016, arXiv e-prints, p. arXiv:1612.05245

Watson C. L., Henden A. A., Price A., 2006, Society for Astronomical Sciences Annual Symposium, 25, 47

Wenger M., et al., 2000, A\&AS, 143, 9

West A. A., Hawley S. L., Bochanski J. J., Covey K. R., Reid I. N., Dhital S., Hilton E. J., Masuda M., 2008, AJ, 135, 785

Wright E. L., et al., 2010a, AJ, 140, 1868

Wright E. L., et al., 2010b, AJ, 140, 1868

York D. G., et al., 2000, AJ, 120, 1579 
APPENDIX A: CONFIGURATION FILES

Table A1: Configuration parameters for SExTRACTOR.

\begin{tabular}{|c|c|}
\hline \multicolumn{2}{|c|}{ Catalog } \\
\hline CATALOG_NAME & test.cat \\
\hline CATALOG_TYPE & FITS_LDAC \\
\hline PARAMETERS_NAME & default.param \\
\hline \multicolumn{2}{|c|}{ Extraction } \\
\hline DETECT_TYPE & $\overline{\mathrm{CCD}}$ \\
\hline DETECT_MINAREA & 3 \\
\hline DETECT_MAXAREA & 0 \\
\hline THRESH_TYPE & RELATIVE \\
\hline DETECT_THRESH & 1.5 \\
\hline ANALYSIS_THRESH & 1.5 \\
\hline FILTER & $\mathrm{Y}$ \\
\hline FILTER_NAME & gauss_2.5_5x5.conv \\
\hline FILTER_THRESH & - \\
\hline DEBLEND_NTHRESH & 32 \\
\hline DEBLEND_MINCONT & 0.0005 \\
\hline CLEAN & $\mathrm{Y}$ \\
\hline CLEAN_PARAM & 1.0 \\
\hline MASK_TYPE & CORRECT \\
\hline \multicolumn{2}{|c|}{ WEIGHTing } \\
\hline WEIGHT_TYPE & MAP_WEIGHT \\
\hline WEIGHT_IMAGE & weight.fits \\
\hline WEIGHT_GAIN & $\mathrm{Y}$ \\
\hline WEIGHT_THRESH & - \\
\hline \multicolumn{2}{|c|}{$\begin{array}{l}\text { FLAGging } \\
\text { Photometry }\end{array}$} \\
\hline PHOT APERTURFS & 202530 \\
\hline PHOT_AUTOPARAMS & $\begin{array}{r}20,20,30 \\
2.5,3.5\end{array}$ \\
\hline PHOT_PETROPARAMS & $2.0,3.5$ \\
\hline PHOT_AUTOAPERS & $0.0,0.0$ \\
\hline PHOT_FLUXFRAC & 0.5 \\
\hline SATUR_LEVEL & 62000.0 \\
\hline SATUR_KEY & DUMMY \\
\hline MAG_ZEROPOINT & 0.0 \\
\hline MAG_GAMMA & 4.0 \\
\hline GAIN & 0.0 \\
\hline GAIN_KEY & GAIN \\
\hline PIXEL_SCALE & 0.0 \\
\hline \multicolumn{2}{|c|}{ Star/Galaxy Separation } \\
\hline SEEING_FWHM & 0.8 \\
\hline STARNNW_NAME & default.nnw \\
\hline \multicolumn{2}{|c|}{ Background } \\
\hline BACK_TYPE & AUTO \\
\hline BACK_VALUE & 0.0 \\
\hline BACK_SIZE & 64 \\
\hline BACK_FILTERSIZE & 3 \\
\hline BACKPHOTO_TYPE & GLOBAL \\
\hline BACKPHOTO_THICK & 24 \\
\hline BACK_FILTTHRESH & 0.0 \\
\hline
\end{tabular}


Table A1: Configuration parameters for SExtractor (continued).

\begin{tabular}{|c|c|}
\hline \multicolumn{2}{|r|}{ Check Image } \\
\hline CHECKIMAGE_TYPE & NONE \\
\hline CHECKIMAGE_NAME & check.fits, aper.fits \\
\hline \multicolumn{2}{|r|}{ Memory } \\
\hline MEMORY_OBJSTACK & 3000 \\
\hline MEMORY_PIXSTACK & 300000 \\
\hline MEMORY_BUFSIZE & 1024 \\
\hline \multicolumn{2}{|r|}{ Miscellaneous } \\
\hline VERBOSE_TYPE & NORMAL \\
\hline HEADER_SUFFIX & head \\
\hline WRITE_XML & $\mathrm{N}$ \\
\hline XML_NAME & sex.xml \\
\hline XSL_URL & file:///usr/share/sextractor/sextractor.xsl \\
\hline NTHREADS & 1 \\
\hline FITS_UNSIGNED & $\mathrm{N}$ \\
\hline INTERP_MAXXLAG & 16 \\
\hline INTERP_MAXYLAG & 16 \\
\hline INTERP_TYPE & NONE \\
\hline \multicolumn{2}{|c|}{ Experimental Stuff } \\
\hline PSF_NAME & 27sfb_barD_0163_ast-red.psf \\
\hline PSF_NMAX & 1 \\
\hline PATTERN_TYPE & RINGS-HARMONIC \\
\hline SOM_NAME & default.som \\
\hline
\end{tabular}


Table A2: Configuration paramerers for PSFEx (continued).

\begin{tabular}{|c|c|}
\hline $\begin{array}{l}\text { CHECKIMAGE_TYPE NONE } \\
\text { CHECKIMAGE_NAME } \\
\text { CHECKIMAGE_CUBE N }\end{array}$ & chi.fits,proto.fits,samp.fits,resi.fits,snap.fits \\
\hline \multicolumn{2}{|c|}{ Miscellaneous } \\
\hline PSF_DIR & - \\
\hline PSF_SUFFIX &.$p s f$ \\
\hline VERBOSE_TYPE & NORMAL \\
\hline WRITE_XML & $\mathrm{Y}$ \\
\hline XML_NAME & psfex.xml \\
\hline XSL_URL & file:///usr/share/psfex/psfex.xsl \\
\hline NTHREADS & 4 \\
\hline
\end{tabular}


Table A3. Parameters obtained from SExtractor and PSFEx.

\begin{tabular}{|c|c|c|}
\hline NUMBER & Running object number & \\
\hline ALPHA_J2000 & Right Ascension of barycenter (J2000) & [deg] \\
\hline DELTA_J2000 & Declination of barycenter (J2000) & {$[\mathrm{deg}]$} \\
\hline XPSF_IMAGE & $\mathrm{X}$ coordinate from PSF-fitting & [pixel] \\
\hline YPSF_IMAGE & Y coordinate from PSF-fitting & [pixel] \\
\hline ERRAPSF_IMAGE & PSF RMS position error along major axis & [pixel] \\
\hline ERRBPSF_IMAGE & PSF RMS position error along minor axis & [pixel] \\
\hline ERRTHETAPSF_IMAGE & PSF error ellipse position angle $(\mathrm{CCW} / \mathrm{x})$ & [deg] \\
\hline XWIN_IMAGE & Windowed position estimate along $\mathrm{x}$ & [pixel] \\
\hline YWIN_IMAGE & Windowed position estimate along y & [pixel] \\
\hline ERRAWIN_IMAGE & RMS windowed position error along major axis & [pixel] \\
\hline ERRBWIN_IMAGE & RMS windowed position error along minor axis & [pixel] \\
\hline ERRTHETAWIN_IMAGE & Windowed error ellipse position angle $(\mathrm{CCW} / \mathrm{x})$ & [deg] \\
\hline FLUX_AUTO & Flux within a Kron-like elliptical aperture & [count] \\
\hline FLUXERR_AUTO & RMS error for AUTO flux & [count] \\
\hline MAG_AUTO & Kron-like elliptical aperture magnitude & {$[\mathrm{mag}]$} \\
\hline MAGERR_AUTO & RMS error for AUTO magnitude & [mag] \\
\hline FLUX_APER & Flux vector within fixed circular aperture(s) & [count] \\
\hline FLUXERR_APER & RMS error vector for aperture flux(es) & [count] \\
\hline MAG_APER & Fixed aperture magnitude vector & {$[\mathrm{mag}]$} \\
\hline MAGERR_APER & RMS error vector for fixed aperture magnitude & {$[\mathrm{mag}]$} \\
\hline FLUX_PSF & Flux from PSF-fitting & [count] \\
\hline FLUXERR_PSF & RMS flux error for PSF-fitting & [count] \\
\hline MAG_PSF & Magnitude from PSF-fitting & {$[\mathrm{mag}]$} \\
\hline MAGERR_PSF & RMS magnitude error from PSF-fitting & [mag] \\
\hline FWHM_IMAGE & FWHM assuming a gaussian core & [pixel] \\
\hline ELONGATION & $\begin{array}{l}\text { A_IMAGE/B_IMAGE (where A_IMAGE is the profile RMS along the major } \\
\text { axis and B_IMAGE is the profile RMS along the minor axis) }\end{array}$ & \\
\hline ELLIPTICITY & $\begin{array}{l}1 \text { - B_IMAGE/A_IMAGE(where A_IMAGE is the profile RMS along the major } \\
\text { axis and B_IMAGE is the profile RMS along the minor axis) }\end{array}$ & \\
\hline FLUX_RADIUS & Fraction-of-light radii & [pixel] \\
\hline FLAGS & Extraction flags & \\
\hline FLAGS_WEIGHT & Weighted extraction flags & \\
\hline SPREAD_MODEL & Spread parameter from model-fitting & \\
\hline SPREADERR_MODEL & Spread parameter error from model-fitting & \\
\hline XMODEL_IMAGE & $\mathrm{X}$ coordinate from model-fitting & [pixel] \\
\hline YMODEL_IMAGE & Y coordinate from model-fitting & [pixel] \\
\hline ERRAMODEL_IMAGE & RMS error of fitted position along major axis & [pixel] \\
\hline ERRBMODEL_IMAGE & RMS error of fitted position along minor axis & [pixel] \\
\hline ERRTHETAMODEL_IMAGE & Error ellipse position angle of fitted position $(\mathrm{CCW} / \mathrm{x})$ & [deg] \\
\hline FLUX_MODEL & Flux from model-fitting & [count] \\
\hline FLUXERR_MODEL & RMS error on model-fitting flux & [count] \\
\hline FLAGS_MODEL & Model-fitting flags & \\
\hline NITER_MODEL & Number of iterations for model-fitting & \\
\hline EXT_NUMBER & FITS extension number & \\
\hline
\end{tabular}


APPENDIX B: CATALOGUE DESCRIPTION 
Table B1. Description of the parameters contained in the detection catalogue.

\begin{tabular}{|c|c|c|}
\hline Parameter & Units & Description \\
\hline $\begin{array}{l}\text { RAJ2000 } \\
\text { DEJ2000 }\end{array}$ & $\begin{array}{l}\operatorname{deg} \\
\operatorname{deg}\end{array}$ & $\begin{array}{l}\text { Right Ascension (J2000). } \\
\text { Declination (J2000). }\end{array}$ \\
\hline $\begin{array}{l}\text { eRA } \\
\text { eDE }\end{array}$ & $\begin{array}{l}\operatorname{arcsec} \\
\operatorname{arcsec}\end{array}$ & $\begin{array}{l}\text { Right Ascension uncertainty }\left(e \_R A J 2000 * \cos \text { DEJ } 2000\right) \text {. } \\
\text { Declination uncertainty. }\end{array}$ \\
\hline ID_source & - & $\begin{array}{l}\text { Unique source identifier. It follows an IAU-style designation of the form } \\
\text { "GTC_OSIRIS_BBI_DR1_JHHMMSS.ss+DDMMSS.s", where "GTC_OSIRIS_BBI_DR1" refers to } \\
\text { the telescope (GTC), the instrument (OSIRIS ), the observing mode (Broad Band Image) and } \\
\text { data release (DR1). The remaining string denotes the J2000 coordinates in sexagesimal format. }\end{array}$ \\
\hline ID_detection & - & $\begin{array}{l}\text { Detection identifier composed by the ID_source and "-n", where } \mathrm{n} \text { is an integer going from } 1 \text { to the } \\
\text { total number of detections of the source. }\end{array}$ \\
\hline Image_url & - & URL access to the FITS image in which the detection has been made. \\
\hline $\begin{array}{l}\text { Xmag_psf } \\
\text { eXmag_psf }\end{array}$ & $\begin{array}{l}\text { mag } \\
\text { mag }\end{array}$ & $\begin{array}{l}\text { PSF calibrated magnitude. } X \text { stands for } g, r, i \text { and } z \text {. } \\
\text { PSF calibrated magnitude error. } X \text { stands for } g, r, i \text { and } z \text {. }\end{array}$ \\
\hline Flag_psf & - & $\begin{array}{l}\text { Flag for PSF calibrated magnitude. } \\
\text { "A" stands for PSF calibrated magnitudes within the interval of magnitudes used for the photometric } \\
\text { calibration. } \\
\text { "B" stands for PSF calibrated magnitudes fainter than the faintest magnitude used for the photo- } \\
\text { metric calibration. } \\
\text { "C" stands for PSF calibrated magnitudes brighter than the brightest magnitude used for the pho- } \\
\text { tometric calibration. }\end{array}$ \\
\hline $\begin{array}{l}\text { Xmag_auto } \\
\text { eXmag_auto }\end{array}$ & $\begin{array}{l}\text { mag } \\
\text { mag }\end{array}$ & $\begin{array}{l}\text { AUTO calibrated magnitude. } X \text { stands for } g, r, i \text { and } z \text {. } \\
\text { AUTO calibrated magnitude error. } X \text { stands for } g, r, i \text { and } z \text {. }\end{array}$ \\
\hline Flag_auto & - & $\begin{array}{l}\text { Flag for AUTO calibrated magnitude. } \\
\text { "A" stands for AUTO calibrated magnitudes within the interval of magnitudes used for the photo- } \\
\text { metric calibration. } \\
\text { "B" stands for AUTO calibrated magnitudes fainter than the faintest magnitude used for the photo- } \\
\text { metric calibration. } \\
\text { "C" stands for AUTO calibrated magnitudes brighter than the brightest magnitude used for the } \\
\text { photometric calibration. }\end{array}$ \\
\hline Xmag_aperY_inst & mag & $\begin{array}{l}\text { Instrumental aperture photometry. } X \text { stands for the four } g, r, i, z \text { and } Y \text { stands for the three apertures } \\
\text { defined in Section } 4 \text {. }\end{array}$ \\
\hline eXmag_aperY_inst & mag & $\begin{array}{l}\text { Instrumental aperture photometric errors. } X \text { stands for } g, r, i, z \text { and } Y \text { stands for the three apertures } \\
\text { defined in Section } 4 .\end{array}$ \\
\hline $\begin{array}{l}\text { Xmag_model_inst } \\
\text { eXmag_model_inst }\end{array}$ & $\begin{array}{l}\text { mag } \\
\text { mag }\end{array}$ & $\begin{array}{l}\text { Instrumental MODEL photometry. } X \text { stands for the four } g, r, i, z \text {. } \\
\text { Instrumental MODEL photometric errors. } X \text { stands for the four } g, r, i, z \text {. }\end{array}$ \\
\hline Elongation & - & Elongation of the detection defined as $A / B$. \\
\hline Ellipticity & - & Ellipticity of the detection defined as $1-B / A$. \\
\hline FWHM & $\operatorname{arcsec}$ & Full width half maximum of the detection. \\
\hline SNR & - & Signal to noise ratio. \\
\hline MJD & $\mathrm{d}$ & Modified Julian Date of the observation. \\
\hline Type & - & $\begin{array}{l}\text { Indicates whether the detection is point-like ("P" or " } \mathrm{P}^{*} \text { ") or extense (" } \mathrm{E}^{\prime} \text { or " } \mathrm{E}^{*} \text { ") as explained in } \\
\text { Section 7.1.2. }\end{array}$ \\
\hline $\mathrm{cl}$ & - & $\begin{array}{l}\text { Point-like coefficient linked to each unique source. It is defined as the ratio between number of } \\
\text { point-like ("P" or "P*") detections and the total number of point-like and extense ("E" or "E*") } \\
\text { classifications of the same source }\left(\text { num } \mathrm{E}_{P} / \text { num } m_{P+E}\right) . c l \text { equal to } 1 \text { indicates that the source has been } \\
\text { always classified as point-like, and } c l \text { equal to } 0 \text { indicates that the source has been always classified } \\
\text { as extense. }\end{array}$ \\
\hline Flag_source & - & Indicates if it is a primary $(\mathrm{p})$ or secondary $(\mathrm{s})$ detection, as defined in Section 7.1.3. \\
\hline
\end{tabular}


Table B2. Description of the parameters contained in the source catalogue.

\begin{tabular}{|c|c|c|}
\hline Parameter & Units & Description \\
\hline RAJ2000 & $\operatorname{deg}$ & Mean weighted Right Ascension (J2000). \\
\hline eRA & $\operatorname{arcsec}$ & $\begin{array}{l}\text { Right Ascension uncertainty }\left(e_{-} R A J 2000 * \cos \mathrm{DEJ} 2000 \text { for sources with unique detection or the stan- }\right. \\
\text { dard deviation of the weighted mean otherwise). }\end{array}$ \\
\hline eDE & $\operatorname{arcsec}$ & $\begin{array}{l}\text { Declination uncertainty ( } e \_D E J 2000 \text { for sources with unique detection or the standard deviation of } \\
\text { the weighted mean otherwise). }\end{array}$ \\
\hline $\mathrm{cl}$ & - & $\begin{array}{l}\text { Point-like coefficient linked to each unique source. It is defined as the ratio between number of } \\
\text { point-like ("P" or "P*") detections and the total number of point-like and extense ("E" or "E*") } \\
\text { classifications of the same source }\left(n u m_{P} / n u m_{P+E}\right) . c l \text { equal to } 1 \text { indicates that the source has been } \\
\text { always classified as point-like, and } c l \text { equal to } 0 \text { indicates that the source has been always classified } \\
\text { as extense. }\end{array}$ \\
\hline ID_source & - & $\begin{array}{l}\text { Unique source identifier. It follows an IAU-style designation of the form } \\
\text { "GTC_OSIRIS_BBI_DR1_JHHMMSS.ss+DDMMSS.s", where "GTC_OSIRIS_BBI_DR1" refers to } \\
\text { the telescope }(G T C \text { ), the instrument (OSIRIS ), the observing mode (Broad Band Image) and } \\
\text { data release (DR1). The remaining string denotes the J2000 coordinates in sexagesimal format. }\end{array}$ \\
\hline ID_detection_Xpsf & - & $\begin{array}{l}\text { Detection identifier of the PSF photometry in the } X \text { band composed by the ID_source and "_n", } \\
\text { where } \mathrm{n} \text { corresponds with the n-th detection of the source in the source catalogue. } X \text { stands for } g \text {, } \\
r, i \text { and } z \text {. }\end{array}$ \\
\hline Image_url_Xpsf & - & $\begin{array}{l}\text { URL access to the FITS image in which the PSF detection has been made. } X \text { stands for } g, r, i \text { and } \\
z \text {. }\end{array}$ \\
\hline $\begin{array}{l}\text { Xmag_psf } \\
\text { eXmag_psf }\end{array}$ & $\begin{array}{l}\text { mag } \\
\text { mag }\end{array}$ & $\begin{array}{l}\text { PSF calibrated magnitude. } X \text { stands for } g, r, i \text { and } z \text {. } \\
\text { PSF calibrated magnitude error. } X \text { stands for } g, r, i \text { and } z \text {. }\end{array}$ \\
\hline Flag_Xpsf & - & $\begin{array}{l}\text { Flag for PSF calibrated magnitude. } X \text { stands for } g, r, i \text { and } z \text {. } \\
\text { "A" stands for PSF calibrated magnitudes within the interval of magnitudes used for the photometric } \\
\text { calibration. } \\
\text { "B" stands for PSF calibrated magnitudes fainter than the faintest magnitude used for the photo- } \\
\text { metric calibration. } \\
\text { "C" stands for PSF calibrated magnitudes brighter than the brightest magnitude used for the pho- } \\
\text { tometric calibration. }\end{array}$ \\
\hline ID_detection_Xauto & - & $\begin{array}{l}\text { Detection identifier of the AUTO photometry in the } X \text { band composed by the ID_source and "-n", } \\
\text { where } \mathrm{n} \text { is an integer going from } 1 \text { to the total number of detections of the source in the source } \\
\text { catalogue. } X \text { stands for } g, r, i \text { and } z \text {. }\end{array}$ \\
\hline Image_url_Xauto & - & $\begin{array}{l}\text { URL access to the FITS image in which the AUTO detection has been made. } X \text { stands for } g, r, i \\
\text { and } z \text {. }\end{array}$ \\
\hline $\begin{array}{l}\text { Xmag_auto } \\
\text { eXmag_auto }\end{array}$ & $\begin{array}{l}\text { mag } \\
\text { mag }\end{array}$ & $\begin{array}{l}\text { AUTO calibrated magnitude. } X \text { stands for } g, r, i \text { and } z \text {. } \\
\text { AUTO calibrated magnitude error. } X \text { stands for } g, r, i \text { and } z \text {. }\end{array}$ \\
\hline Flag_Xauto & - & $\begin{array}{l}\text { Flag for AUTO calibrated magnitude. } X \text { stands for } g, r, i \text { and } z \text {. } \\
\text { "A" stands for AUTO calibrated magnitudes within the interval of magnitudes used for the photo- } \\
\text { metric calibration. } \\
\text { "B" stands for AUTO calibrated magnitudes fainter than the faintest magnitude used for the photo- } \\
\text { metric calibration. } \\
\text { "C" stands for AUTO calibrated magnitudes brighter than the brightest magnitude used for the } \\
\text { photometric calibration. }\end{array}$ \\
\hline MJD_Xauto & $\mathrm{d}$ & Modified Julian Date of the observation of the AUTO photometry. $X$ stands for $g, r, i$ and $z$. \\
\hline
\end{tabular}


APPENDIX C: TABLE OF COOL DWARF CANDIDATES 
Table C1. OSIRIS and Gaia DR2 identifiers, and effective temperatures of the 49 cool dwarf candidates (“*” identifies the secondary component of the close $\mathrm{K}+\mathrm{M}$ binary system) plus the late-type binary ("***" and "***" identify the primary and the secondary components, respectively) found in this work.

\begin{tabular}{|c|c|c|}
\hline ID & Gaia DR2 source_id & $T_{e f f}[\mathrm{~K}]$ \\
\hline GTC_OSIRIS_BBI_DR1_J095528.33+694328.2 & 1070554378447970176 & 3000.0 \\
\hline GTC_OSIRIS_BBI_DR1_J095533.01+694339.1 & 1070554378447969920 & 3200.0 \\
\hline GTC_OSIRIS_BBI_DR1_J095612.91+693729.9 & 1070549220190318464 & 3100.0 \\
\hline GTC_OSIRIS_BBI_DR1_J111433.38-302022.4 & 5404305499712757888 & 3100.0 \\
\hline GTC_OSIRIS_BBI_DR1_J111449.77-302006.9 & 5404305121755637632 & 3400.0 \\
\hline GTC_OSIRIS_BBI_DR1_J152215.23+285622.0 & 1272146188971317632 & 3200.0 \\
\hline GTC_OSIRIS_BBI_DR1_J152224.31+290123.5 & 1272152923480070144 & 3400.0 \\
\hline GTC_OSIRIS_BBI_DR1_J154542.83-001823.1 & 4404496211953819392 & 3300.0 \\
\hline GTC_OSIRIS_BBI_DR1_J164751.11+715102.1 & 1653530664857859456 & 3100.0 \\
\hline GTC_OSIRIS_BBI_DR1_J164823.16+714939.3 & 1653529973366993792 & 3300.0 \\
\hline GTC_OSIRIS_BBI_DR1_J170533.87+112345.0 & 4444960919915917440 & 3100.0 \\
\hline GTC_OSIRIS_BBI_DR1_J181603.76+691300.4 & 2259804228271304192 & 3300.0 \\
\hline GTC_OSIRIS_BBI_DR1_J194704.87+313659.8 & 2034508278496366208 & 3100.0 \\
\hline GTC_OSIRIS_BBI_DR1_J203115.87+410311.3 & 2067801078262984960 & 3200.0 \\
\hline GTC_OSIRIS_BBI_DR1_J203116.85+410028.4 & 2067797917166997248 & 3400.0 \\
\hline GTC_OSIRIS_BBI_DR1_J203118.11+405343.5 & 2067794962230400000 & 3100.0 \\
\hline GTC_OSIRIS_BBI_DR1_J203146.14+412246.4 & 2067829356329503616 & 2900.0 \\
\hline GTC_OSIRIS_BBI_DR1_J203146.23+411437.0** & 2067827608276334848 & \\
\hline GTC_OSIRIS_BBI_DR1_J203146.19+411437.6*** & 2067827608279535744 & \\
\hline GTC_OSIRIS_BBI_DR1_J203151.94+410902.4 & 2067822484380101888 & 3200.0 \\
\hline GTC_OSIRIS_BBI_DR1_J203152.80+412429.0 & 2067829493768462464 & 3400.0 \\
\hline GTC_OSIRIS_BBI_DR1_J203159.58+412624.2 & 2067835511019676544 & 3700.0 \\
\hline GTC_OSIRIS_BBI_DR1_J203204.11+410327.8 & 2067773968429422976 & 3200.0 \\
\hline GTC_OSIRIS_BBI_DR1_J203204.73+411135.0 & 2067824064928106880 & 3300.0 \\
\hline GTC_OSIRIS_BBI_DR1_J203213.30+412156.6 & 2067834338490023552 & 3600.0 \\
\hline GTC_OSIRIS_BBI_DR1_J203218.88+412303.6 & 2067834166691344896 & 3500.0 \\
\hline GTC_OSIRIS_BBI_DR1_J203228.12+410853.8 & 2067776476690427008 & 3800.0 \\
\hline GTC_OSIRIS_BBI_DR1_J203229.46+410025.6 & 2067773040716423680 & 3300.0 \\
\hline GTC_OSIRIS_BBI_DR1_J203240.40+410329.6 & 2067774689983932032 & 3500.0 \\
\hline GTC_OSIRIS_BBI_DR1_J203255.73+411428.9 & 2067782901961590528 & 3200.0 \\
\hline GTC_OSIRIS_BBI_DR1_J203302.89+412236.0 & 2067831967669718144 & 3500.0 \\
\hline GTC_OSIRIS_BBI_DR1_J203307.46+410420.9 & 2067777855378562432 & 2400.0 \\
\hline GTC_OSIRIS_BBI_DR1_J203317.38+411906.9 & 2067785002201372032 & 3200.0 \\
\hline GTC_OSIRIS_BBI_DR1_J203323.48+412040.5 & 2067785135346315136 & 3000.0 \\
\hline GTC_OSIRIS_BBI_DR1_J203324.41+410751.7* & 2067777782367558784 & 3300.0 \\
\hline GTC_OSIRIS_BBI_DR1_J203341.70+405844.3 & 2067763802245107584 & 3300.0 \\
\hline GTC_OSIRIS_BBI_DR1_J203344.35+412131.5 & 2067878078436914560 & 2800.0 \\
\hline GTC_OSIRIS_BBI_DR1_J203353.39+410013.9 & 2067764244624468736 & 3100.0 \\
\hline GTC_OSIRIS_BBI_DR1_J203402.48+413937.6 & 2067935291698824064 & 3200.0 \\
\hline GTC_OSIRIS_BBI_DR1_J203403.33+410246.1 & 2067765893892779008 & 3300.0 \\
\hline GTC_OSIRIS_BBI_DR1_J203403.60+412450.4 & 2067877150723292288 & 3200.0 \\
\hline GTC_OSIRIS_BBI_DR1_J203404.41+405530.4 & 2067760087096005760 & 3200.0 \\
\hline GTC_OSIRIS_BBI_DR1_J203408.50+411954.7 & 2067874543679715200 & 3000.0 \\
\hline GTC_OSIRIS_BBI_DR1_J203415.44+405716.3 & 2067761637577789312 & 3000.0 \\
\hline GTC_OSIRIS_BBI_DR1_J203416.90+411944.7 & 2067875776335180800 & 3000.0 \\
\hline GTC_OSIRIS_BBI_DR1_J203425.02+405820.6 & 2067761598925476352 & 3600.0 \\
\hline GTC_OSIRIS_BBI_DR1_J203430.63+411138.3 & 2067860967287122816 & 3100.0 \\
\hline GTC_OSIRIS_BBI_DR1_J203434.94+412553.5 & 2067876708344798336 & 3600.0 \\
\hline GTC_OSIRIS_BBI_DR1_J203446.65+405656.5 & 2064759279345218304 & 3300.0 \\
\hline GTC_OSIRIS_BBI_DR1_J203509.14+415510.5 & 2067916801861913984 & 3200.0 \\
\hline GTC_OSIRIS_BBI_DR1_J203515.96+410601.1 & 2064855486612035584 & 3200.0 \\
\hline
\end{tabular}

